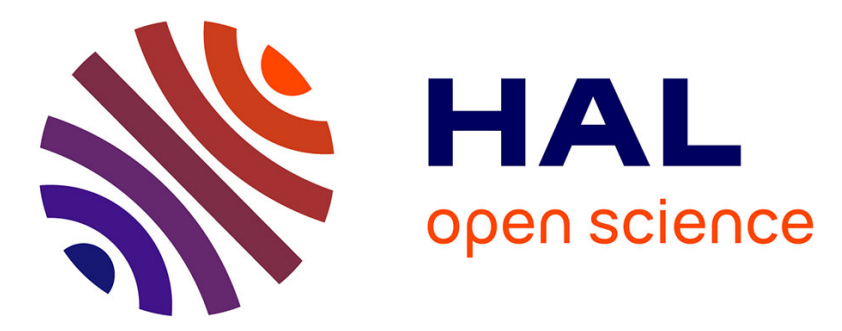

\title{
Hotelling Games on Networks: Efficiency of Equilibria
}

Gaëtan Fournier, Marco Scarsini

\section{To cite this version:}

Gaëtan Fournier, Marco Scarsini. Hotelling Games on Networks: Efficiency of Equilibria. 2014. halshs-00983085

\section{HAL Id: halshs-00983085 https://shs.hal.science/halshs-00983085}

Submitted on 24 Apr 2014

HAL is a multi-disciplinary open access archive for the deposit and dissemination of scientific research documents, whether they are published or not. The documents may come from teaching and research institutions in France or abroad, or from public or private research centers.
L'archive ouverte pluridisciplinaire HAL, est destinée au dépôt et à la diffusion de documents scientifiques de niveau recherche, publiés ou non, émanant des établissements d'enseignement et de recherche français ou étrangers, des laboratoires publics ou privés. 


\section{Documents de Travail du Centre d'Economie de la Sorbonne}

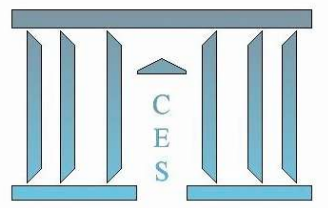

Hotelling Games on Networks: Efficiency of Equilibria

Gaëtan FoURNIER, Marco SCARSINI

2014.33

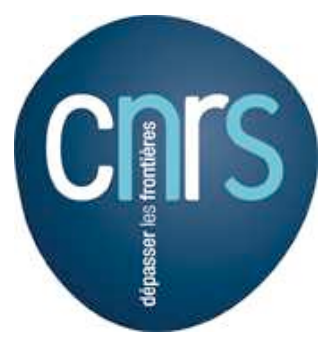

Maison des Sciences Économiques, 106-112 boulevard de L'Hôpital, 75647 Paris Cedex 13 


\title{
Hotelling Games on Networks: Efficiency of Equilibria*
}

\author{
Gaëtan Fournier \\ Centre d'Economie de la Sorbonne \\ Université Paris 1 Panthéon-Sorbonne \\ 106-112 Boulevard de l'Hôpital \\ 75647 Paris Cedex 13 \\ France \\ fournier.gtn@gmail.com \\ Marco Scarsini ${ }^{\dagger}$ \\ Engineering and System Design Pillar \\ Singapore University of Technology and Design \\ 20 Dover Drive \\ Singapore 138682 \\ marco_scarsini@sutd.edu.sg
}

April 6, 2014

\footnotetext{
*Partially supported by PRIN 20103S5RN3, SRG ESD 2012 044, and MOE2013-T2-1-158.

${ }^{\dagger}$ On leave from Dipartimento di Economia e Finanza, LUISS, Roma. This work was carried out while this author was a member of GNAMPA-INdAM.
} 


\begin{abstract}
We consider a Hotelling game where a finite number of retailers choose a location, given that their potential customers are distributed on a network. Retailers do not compete on price but only on location, therefore each consumer shops at the closest store. We show that when the number of retailers is large enough, the game admits a pure Nash equilibrium and we construct it. We then compare the equilibrium cost bore by the consumers with the cost that could be achieved if the retailers followed the dictate of a benevolent planner. We perform this comparison in term of the induced price of anarchy, i.e., the ratio of the worst equilibrium cost and the optimal cost, and the induced price of stability, i.e., the ratio of the best equilibrium cost and the optimal cost. We show that, asymptotically in the number of retailers, these ratios are two and one, respectively.
\end{abstract}

JEL Classification: C72, R30, R39.

Keywords: Induced price of anarchy, induced price of stability, location games on networks, pure equilibria, large games. 


\section{Introduction}

In his famous seminal paper Hotelling (1929) considers duopoly models with differentiated products. The article is extremely rich in modeling, motivation, and examples in different areas. Among the different models in the paper, the one that came to be known as the Hotelling's model, involves two retailers along a road (a segment), who can decide where to locate their shops. Consumers are assumed to be uniformly distributed along the road and their preference is to patronize the nearest shop. Although it would be socially optimal that the two retailers set up their shops respectively at $1 / 4$ and $3 / 4$ of the distance from one end of the road, this behavior would not give rise to an equilibrium and would induce profitable deviations for each of the players. In equilibrium both retailers set shop in the middle of the road, giving rise to a suboptimal situation for the consumers, who now, on average, have to travel twice as much as in the social optimum to reach their preferred store. Although not expressed in the language of game theory (the paper by von Neumann (1928) had just appeared and John Nash was one year old), the paper contains an insightful analysis of the tension between social optimum and strategic behavior of agents. Hotelling emphasizes how differentiation of products can refer to features different from geographical distance and mentions even some applications in political science, where the product becomes the program that candidates propose to their voters. He shows how the principle of minimum differentiation applies in different contexts.

Hotelling's model has been generalized and modified in many directions, as will be described below. In most of the existing literature the set of possible actions for the retailers is very simple, e.g, a segment or a circle. In this paper we consider a model where consumers are uniformly distributed on a network. A finite number of retailers can set shop anywhere they want on this network. They all sell the same product and their decision variable is only the location. This model applies for instance to shops that sell products whose price is exogenously determined, for instance newsstands, pharmacies, franchises of different types of services and products, e.g., mobile telephones, brand clothes, etc.

Consumers are assumed to be homogeneous in the sense that they buy the same quantity of goods and, given the uniformity of prices, they shop at the closest store. We assume that the cost for a consumer is proportional to the distance she has to travel to do her shopping. The network where consumers are distributed is very general: connectedness is not assumed, only finite length. We model the situation as a game where the payers are the retailers, their actions are the location they choose on the network, and their payoff is the amount of consumers they can convince to shop at their store. We concentrate our attention on the existence of pure equilibria.

We compare the outcome of an equilibrium of the game to the situation where a planner makes optimal decisions for everybody. Similar comparisons are usually performed through the price of anarchy, i.e., the ratio between the total socially optimal payoff and the total payoff induced by the worst equilibrium. A different 
comparison is obtained by considering the price of stability, that is, ratio between the total socially optimal payoff and the total payoff induced by the best equilibrium.

Since the retailers split the total mass of consumers among themselves, the game is fixed-sum, so the price of anarchy is not at all informative. Nevertheless, something interesting can be obtained if, instead of taking the retailers' viewpoint, we examine the situation from the consumers' point of view. It is clear that the retailers' behavior affects the cost incurred by the consumers, that is, the distance they have to travel to reach the closest shop. Hence we consider the ratio between the worst total consumer cost that an equilibrium can induce and the optimal total cost that a benevolent planner can achieve. We call this new measure of efficiency induced price of anarchy, to emphasize the fact that it does not concern the players of the games, but rather the passive subjects (customers). In an analogous way, we define the induced price of stability, that is, the ratio between the best total consumer cost that an equilibrium can induce and the optimal total cost that a benevolent planner can achieve.

The main results of the paper concern existence of equilibria and the asymptotic value of the induced prices of anarchy and stability. In particular we show that, whatever the network, a pure Nash equilibrium of the game exists when the number of players is large enough, and we construct such an equilibrium. For specific networks, more precise results hold. For instance, when the network is a star, we show that when the number of players lies in a specific interval, then the game does not admit pure equilibria. For any number of players outside this interval, equilibria exist and we can say when they are unique.

Moreover we show that the induced price of anarchy is asymptotically 2, no matter what the network is. Therefore, when the number of players is large, the induced price of anarchy becomes close to the one obtained when consumers are distributed on a circle. We also show that the induced price of stability is asymptotically 1 , that is, that efficiency can approximately be achieved in equilibrium, when the number of retailers is large. This shows that, when the number of players is large, the best role that a planner can play in achieving efficiency is to propose a good equilibrium to the players, given that the game is zero-sum, so retailers have no reason to prefer one equilibrium over another.

The results on the induced price of anarchy and on the induced price of stability are proved using majorization techniques (see, e.g. Marshall, Olkin, and Arnold, 2011).

\section{Review of the literature}

Several generalizations and variations of Hotelling's model have been considered in the literature. Here we cite only a small fraction of the contributions to the topic. Anderson, de Palma, and Thisse (1992) contains a nice survey of the literature up to 1992. For a more recent survey the reader is referred to Brenner (2010).

Shortly after Hotelling (1929), both Chamberlin (1933) and Lerner and Singer (1937) criticize Hotelling's results and consider models with more than two firms. 
Eaton and Lipsey (1975) describe a Hotelling-type model with an arbitrary number of players, different possible structures of the space where retailers can locate, and different distributions of the customers. They show how the principle of minimum differentiation holds only under strong hypotheses. d'Aspremont, Gabszewicz, and Thisse (1979) show that in a model where duopolists compete on price the principle of minimum differentiation does not hold. de Palma, Ginsburgh, Papageorgiou, and Thisse (1985) show that Hotelling's principle of minimum differentiation holds when products and consumers are heterogeneous.

Osborne and Pitchik (1986) study mixed equilibria of the Hotelling game and Osborne and Pitchik (1987) consider a two-stage model where firms choose location in the first stage and price in the second. Economides (1993) studies a model with more than two firms, proves the existence of an equilibrium in prices, and shows that no subgame perfect equilibrium exists in a two stage model where location is chosen in the first stage and price in the second. Bester, de Palma, Leininger, Thomas, and von Thadden (1996) consider a two-firm two-stage model with quadratic transportation costs and prove that infinite mixed equilibria exist.

Salop (1979) considers a model with two different industries where the technical corner difficulties of Hotelling's model are eliminated by assuming that the consumers are distributed on a circle. Lederer and Hurter (1986) consider a model where firms are different and consumers are non uniformly distributed on the plane. Aoyagi and Okabe (1993) look at a bidimensional market and, with the help of simulation, relate the existence of equilibria and their properties to the shape of the market. Eiselt and Laporte (1993) model the case of three firms on a tree. Anderson, Goeree, and Ramer (1997) study a model with two firms and nonuniform distribution of consumers. They find conditions on this distribution for existence of equilibria. Irmen and Thisse (1998) develop a duopoly model where differentiation can happen across several dimensions.

Mavronicolas, Monien, Papadopoulou, and Schoppmann (2008) and Feldmann, Mavronicolas, and Monien (2009) consider a Hotelling model on graphs where firms can locate only on the vertices of the graph. Soetevent (2010) extends Hotelling's model of price competition with quadratic transportation costs from a line to graphs. Pálvölgyi (2011) considers Hotelling games on graphs. His approach is quite close to the one used in this paper, but, unfortunately, his results are not accurate.

The price of anarchy was introduced by Koutsoupias and Papadimitriou (1999) and the name was coined by Papadimitriou (2001). Several authors have studied this topic in different contexts: routing games (see, e.g., Roughgarden and Tardos, 2002, 2004, Correa, Schulz, and Stier-Moses, 2004, 2007, 2008), cost sharing (see, e.g., Moulin, 2008, Epstein, Feldman, and Mansour, 2009, Hoefer, 2011), auctions (see, e.g., Moulin, 2010), coalition formation (see, e.g., Immorlica, Markakis, and Piliouras, 2013), health care (see, e.g., Knight and Harper, 2013), games with incomplete information (see, e.g., Roughgarden, 2012), etc. The price of stability was introduced by Schulz and Stier Moses (2003). We are not aware of any previous use of the induced price of anarchy or the induced price of stability. 


\section{Organization of the paper}

In Section 2 the model is introduced. Section 3 provides some general results. Several examples are considered in Section 4. All the proofs can be found in the Appendix.

\section{The model}

\subsection{The network}

A planar curve (or simply a curve) is a continuous mapping $\phi:[0,1] \rightarrow \mathbb{R}^{2}$. Its length $\lambda(\phi)$ is defined as

$$
\lambda(\phi):=\sup _{m \in \mathbb{N}}\left\{\sum_{i=1}^{m}\left\|\phi\left(t_{i}\right)-\phi\left(t_{i-1}\right)\right\|: 0=t_{0}<t_{1}<\cdots<t_{m}=1\right\}
$$

A curve is called simple if it is injective and rectifiable if it has finite length.

Consider a planar graph $(V, E)$, where $V \subset \mathbb{R}^{2}$ is a finite set of vertices and $E$ is a finite set of edges. We define the network $S$ to be the planar embedding of the graph and we identify each edge $e \in E$ with the corresponding rectifiable curve of the planar embedding, which, with an abuse of language, we still call $e$. If $e$ connects the vertices $u, v$, then we sometime use the notation $[u, v]$ for $e$ and $(u, v)$ for $[u, v] \backslash\{u, v\}$.

Given two points $x, y \in S$ we define their distance $d(x, y)$ as the length of the shortest path in $S$ that connects them. The distance $d(x, y)$ is infinite if $S$ is not connected and $x$ and $y$ belong to different connected components of the network.

The network $S$ is then a Borel set, endowed with the measure generated by the length. We will use the same symbol $\lambda$ for this measure. We define

$$
L:=\lambda(S)=\sum_{e \in E} \lambda(e)
$$

We call leaf a vertex $v \in V$ such that $\operatorname{degree}(v)=1$. The network $S$ generated by a graph $(V, E)$ is equivalent to a network generated by a sub-graph whose vertices have degree different from 2. This subgraph can be obtained by performing this simple operation: whenever the vertex $u$ has degree two, delete it and replace $[v, u]$ and $[u, w]$ with $[v, w]$ so that $\lambda([v, w])=\lambda([v, u])+\lambda([u, w])$. Therefore we will always assume that $V$ contains no vertices of degree 2 . We extend the definition of the function degree from $V$ to $S$ by assuming that degree $(x)=2$ for all $x \in S \backslash V$.

\subsection{The game}

We consider a situation where each of $n$ retailers has to decide where to locate her shop on a network, given that an uncountable population of consumers is uniformly distributed on the network and each consumer patronizes a shop in the closest 
location, where ties will be solved as follows. Consider the set $A$ of consumers that are equally distant from $k$ different locations having each at least one shop. Then we assume that for each of these $k$ locations, $\lambda(A) / k$ consumers go to that location. Moreover if one of the $k$ locations has $h$ shops, then a fraction $\lambda(A) / h k$ patronizes each shop of this location.

Basically the network is decomposed into domains of attraction of different retailers's locations and then within each domain of attraction retailers in the same location split the consumers equally. Some parts of the network can belong to different domains of attraction, as the following example shows.

Example 2.1. Consider the network in Figure 1 with seven players. Assume that $\lambda\left(e_{4}\right)=\lambda\left(e_{5}\right)$ and that two retailers are located in $u$ and five retailers are located in $v$. All the points in $e_{8}$ are equally distant from $u$ and $v$. Therefore the retailers in $u$ jointly attract all the consumers on the red edges plus half of the consumers on $e_{8}$. The retailers in $v$ jointly attract all the consumers on the green edges plus the remaining half of the consumers on $e_{8}$. That is, each player in $u$ attracts the following quantity of consumers

$$
\frac{1}{2}\left(\lambda\left(e_{1}\right)+\lambda\left(e_{2}\right)+\lambda\left(e_{3}\right)+\lambda\left(e_{4}\right)+\frac{1}{2} \lambda\left(e_{8}\right)\right)
$$

and each player in $v$ attracts the following quantity of consumers

$$
\frac{1}{5}\left(\lambda\left(e_{5}\right)+\lambda\left(e_{6}\right)+\lambda\left(e_{7}\right)+\frac{1}{2} \lambda\left(e_{8}\right)\right) .
$$

This example shows that the situation of a Hotelling game on a general network is more complicated than the classical case of a game on a circle or a segment. The fact that a set of positive measure may be equidistant from two points imposes some extra care in the definition of the domain of attraction of retailers.

\section{FIGURE 1 ABOUT HERE}

We model this as a normal form game with a finite set $N_{n}=\{1, \ldots, n\}$ of players having all the same action set $S$. When there is no risk of confusion we will just write $N$ instead of $N_{n}$. Each player represents a retailer, whose payoff is the measure of the set of consumers who shop at her store. In order to formally define the players' payoffs, we need to introduce the following quantities. Given $\boldsymbol{x}=\left(x_{1}, \ldots, x_{n}\right) \in S^{n}$, call $X(\boldsymbol{x})$ the set of all $y \in S$ for which there exists $i \in N_{n}$ such that $y=x_{i}$. In general $\operatorname{card}(X(\boldsymbol{x})) \leq n$, since several players can chose the same location. For $K \subset X(\boldsymbol{x})$ define

$$
\begin{aligned}
& Y_{K}=\left\{y \in S: d\left(y, x_{i}\right)=d\left(y, x_{j}\right) \text { for all } x_{i}, x_{j} \in K\right. \\
& \text { and } \left.d\left(y, x_{i}\right)<d\left(y, x_{\ell}\right) \text { for all } x_{i} \in K, x_{\ell} \notin K\right\} .
\end{aligned}
$$


The payoff of player $i \in N_{n}$ under the strategy profile $\boldsymbol{x}$ is

$$
\rho_{i}(\boldsymbol{x})=\frac{1}{\operatorname{card}\left(\left\{j \in N_{n}: x_{j}=x_{i}\right\}\right)} \sum_{K \subset N_{n}} \mathbb{1}_{x_{i} \in K} \frac{\lambda\left(Y_{K}\right)}{\operatorname{card}(K)} .
$$

The above defined game is called Hotelling game on $S$ and is denoted by $\mathscr{H}\left(N_{n}, S, \boldsymbol{\rho}\right)$, where $\boldsymbol{\rho}=\left(\rho_{1}, \ldots, \rho_{n}\right)$. The set of all its Nash equilibria is denoted by $\mathscr{E}(\mathscr{H})$.

Consider a game $\mathscr{H}\left(N_{n}, S, \boldsymbol{\rho}\right)$. For $\boldsymbol{x}=\left(x_{1}, \ldots, x_{n}\right) \in S^{n}$ and $y \in S$ define

$$
d(\boldsymbol{x}, y):=\min _{i \in\{1, \ldots, n\}}\left(d\left(x_{i}, y\right)\right) .
$$

If $S$ is not connected, then it can happen that no $x_{i}$ is in the same component as $y$, and therefore $d(\boldsymbol{x}, y)=\infty$.

The social cost $\sigma(\boldsymbol{x})$ is defined as

$$
\sigma(\boldsymbol{x}):=\int_{S} d(\boldsymbol{x}, y) \mathrm{d} y .
$$

Again, if $S$ is not connected, we can have $\sigma(\boldsymbol{x})=\infty$.

Definition 2.2. Consider a game $\mathscr{H}\left(N_{n}, S, \boldsymbol{\rho}\right)$ that admits a Nash equilibrium. We define

1. the socially optimal cost

$$
\operatorname{Opt}\left(\mathscr{H}\left(N_{n}, S, \boldsymbol{\rho}\right)\right):=\inf _{\boldsymbol{x} \in S^{n}} \sigma(\boldsymbol{x})
$$

2. the worst equilibrium cost

$$
\mathrm{WEq}\left(\mathscr{H}\left(N_{n}, S, \boldsymbol{\rho}\right)\right):=\inf _{\boldsymbol{x} \in \mathscr{E}(\mathscr{H})} \sigma(\boldsymbol{x}),
$$

3. the best equilibrium cost

$$
\operatorname{BEq}\left(\mathscr{H}\left(N_{n}, S, \boldsymbol{\rho}\right)\right):=\sup _{\boldsymbol{x} \in \mathscr{E}(\mathscr{H})} \sigma(\boldsymbol{x}),
$$

4. the induced price of anarchy

$$
\operatorname{IPoA}\left(\mathscr{H}\left(N_{n}, S, \boldsymbol{\rho}\right)\right):=\frac{\text { WEq } \mathscr{H}\left(N_{n}, S, \boldsymbol{\rho}\right)}{\operatorname{Opt} \mathscr{H}\left(N_{n}, S, \boldsymbol{\rho}\right)},
$$

5. the induced price of stability

$$
\operatorname{IPoS}\left(\mathscr{H}\left(N_{n}, S, \boldsymbol{\rho}\right)\right):=\frac{\operatorname{BEq} \mathscr{H}\left(N_{n}, S, \boldsymbol{\rho}\right)}{\operatorname{Opt} \mathscr{H}\left(N_{n}, S, \boldsymbol{\rho}\right)} .
$$

Notice that Opt $\mathscr{H}\left(N_{n}, S, \boldsymbol{\rho}\right)>0$ since $n$ is finite and $\lambda(S)>0$, so there always exists a positive mass of consumers at a strictly positive distance from the closest possible retailer. Therefore both $\operatorname{IPoA}\left(\mathscr{H}\left(N_{n}, S, \boldsymbol{\rho}\right)\right)$ and $\operatorname{IPoS}\left(\mathscr{H}\left(N_{n}, S, \boldsymbol{\rho}\right)\right)$ are well defined. 


\section{General results}

The results in this section are valid for any possible finite network, not necessarily connected. The first theorem shows that Hotelling games always have pure Nash equilibria, provided the number of players is large enough. To be more precise, for every fixed network, there exists a threshold such that, for any number of players above this threshold, the game admits a pure Nash equilibrium.

Theorem 3.1. For an arbitrary $S$, there exists $\bar{n} \in \mathbb{N}$ such that for every $n \geq \bar{n}$, the game $\mathscr{H}\left(N_{n}, S, \boldsymbol{\rho}\right)$ admits a Nash equilibrium.

A similar result was stated and proved by Pálvölgyi (2011). Unfortunately his statement and proof appear to be incorrect.

The next theorem provides a bound for the induced price of anarchy in a Hotelling game. A by-product of this result is that asymptotically the induced price of anarchy cannot exceed 2. As the following sections show, the result holds exactly and not only asymptotically for simple configurations of the network. The same theorem provides an asymptotic result on the price of stability.

Given a graph $(V, E)$, define

$$
\begin{aligned}
V_{I} & =\{v \in V: \operatorname{degree}(v) \geq 3\}, \\
V_{L} & =\{v \in V: \operatorname{degree}(v)=1\}, \\
E_{L} & =\left\{e \in E: e=(v, w), v \in V_{I}, w \in V_{L}\right\}, \\
E_{L L} & =\left\{e \in E: e=(v, w), v, w \in V_{L}\right\}, \\
E_{I} & =\left\{e \in E: e=\left(v, v^{\prime}\right), v, w \in V_{I}\right\} .
\end{aligned}
$$

Theorem 3.2. Suppose that the game $\mathscr{H}\left(N_{n}, S, \boldsymbol{\rho}\right)$ has an equilibrium. Then

(a)

$$
\operatorname{IPoA}\left(\mathscr{H}\left(N_{n}, S, \boldsymbol{\rho}\right)\right) \leq 2+\frac{2 \operatorname{card}\left(E_{I}\right)+\operatorname{card}\left(E_{L}\right)}{n}
$$

(b)

$$
\lim _{n \rightarrow \infty} \operatorname{IPoS}\left(\mathscr{H}\left(N_{n}, S, \boldsymbol{\rho}\right)\right)=1
$$

The interpretation of Theorem 3.2 is that, when the number of retailers is large, if they are left to their own devices and play a bad Nash equilibrium, the outcome of their actions could decrease efficiency by a factor of two, approximately. On the other hand, if a planner cajoles them to play a suitable Nash equilibrium, then efficiency is almost achieved.

Given that the game is zero-sum and symmetric, the retailers have no clear preference of an equilibrium over another, on the other hand, in general, they have no incentive to play a social optimum that is not an equilibrium. The role of the planner is then to propose to the players a profile of strategy that is approximately efficient and, moreover, is stable, i.e., is a Nash equilibrium. 


\section{Examples}

In this section we consider some simple examples of networks and show that in these cases exact results can be obtained.

\subsection{The circle}

In this model consumers are uniformly distributed on the unit circle $C$. This model has been studied by Eaton and Lipsey (1975) and Salop (1979).

Proposition 4.1. For every $n \in \mathbb{N}$ the set of equilibria of the game $\mathscr{H}\left(N_{n}, C, \boldsymbol{\rho}\right)$ is a non-empty polytope.

Proposition 4.2. We have

(a)

$$
\operatorname{IPoA}\left(\mathscr{H}\left(N_{n}, C, \boldsymbol{\rho}\right)\right)= \begin{cases}2 & \text { if } n \text { is even } \\ 2 \frac{n}{n+1} & \text { if } n \text { is odd }\end{cases}
$$

(b) For positive $n$

$$
\operatorname{IPoS}\left(\mathscr{H}\left(N_{n}, C, \boldsymbol{\rho}\right)\right)=1 .
$$

Figures 2, 3, 4 show examples of equilibria on the circle.

FIGURES 2, 3, 4 ABOUT HERE

\subsection{The segment}

The model described in this subsection was studied in details by Eaton and Lipsey (1975) under slightly different assumptions. We consider a Hotelling game on a segment, which, without any loss of generality, is assumed to be $[0,1]$.

Proposition 4.3. Consider the Hotelling game $\mathscr{H}\left(N_{n},[0,1], \boldsymbol{\rho}\right)$.

(a) For $n=2,4,5$ there exists a unique (modulo permutation of players) pure Nash equilibrium (see Figures 5, 6, 7).

(b) For $n=3$, there is no pure Nash equilibrium.

(c) For $n>6$, there is an infinite number of pure Nash equilibria. They form an $(n-5)$-dimensional convex polytope. 


\section{FIGURES 5, 6, 7, 8 ABOUT HERE}

If we call $\eta_{i}=x_{i+4}-x_{i+3}$ then Figure 8 is a example of $n$-player Nash equilibrium.

We now compute the induced price of anarchy and the induced price of stability for Hotelling games on a segment.

Proposition 4.4. We have

(a)

$$
\operatorname{IPoA}\left(\mathscr{H}\left(N_{n},[0,1], \boldsymbol{\rho}\right)\right)= \begin{cases}2 & \text { if } n \text { is even } \\ 2 \frac{n}{n+1} & \text { if } n>3 \text { is odd } .\end{cases}
$$

(b) For $n=2$

$$
\operatorname{IPoS}\left(\mathscr{H}\left(N_{n},[0,1], \boldsymbol{\rho}\right)\right)=\operatorname{IPoA}\left(\mathscr{H}\left(N_{n},[0,1], \boldsymbol{\rho}\right)\right)=2
$$

For $n \geq 4$

$$
\operatorname{IPoS}\left(\mathscr{H}\left(N_{n},[0,1], \boldsymbol{\rho}\right)\right)=\frac{n}{n-2} .
$$

Figures 9, 10, 11, 12 provide examples of social optima, and best and worst equilibria on the segment.

FIGURES 9, 10, 11, 12 ABOUT HERE

\subsection{The star}

In the whole section, we have $k>2$. We consider the case where $S$ is a $\operatorname{star} S_{k}$, that is a network with $k+1$ vertices $\left\{v_{0}, v_{1}, \ldots, v_{k}\right\}$ where for $j \in\{1, \ldots, k\}$ vertex $v_{j}$ is connected to vertex $v_{0}$ and to no other vertex. In the whole section, we assume that the length of the edges $\left[v_{0}, v_{j}\right]$ is the same for all $j$. Without any loss of generality we assume this length to be 1 .

Proposition 4.5. Consider a Hotelling game $\mathscr{H}\left(N_{n}, S_{k}, \boldsymbol{\rho}\right)$.

(a) If $n \leq k$, then a unique equilibrium $\boldsymbol{x}^{*}$ exists where $x_{i}^{*}=v_{0}$ for all $i \in N_{n}$.

(b) If $k<n<3 k-1$, then there is no Nash equilibrium.

(c) If $3 k-1 \leq n \leq 3 k$, there exists a unique equilibrium.

(d) If $3 k+1 \leq n$ then there exists an infinite number of equilibria.

Figures 13, 14, 15 show some examples of equilibria on the star with different numbers of players.

FIGURES 13, 14, 15 ABOUT HERE 


\section{A Proofs}

\section{Majorization}

We introduce some well-known basic concepts in the theory of majorization that will be used in the proof of some results. We refer the reader to Marshall et al. (2011) for an extensive analysis of this topic.

Definition A.1. Given a vector $\boldsymbol{z}=\left(z_{1}, \ldots, z_{n}\right)$, call $z_{[1]} \geq \cdots \geq z_{[n]}$ its decreasing rearrangement. Let $\boldsymbol{x}, \boldsymbol{y} \in \mathbb{R}_{+}^{n}$ be such that

$$
\sum_{i=1}^{n} x_{i}=\sum_{i=1}^{n} y_{i}
$$

and, for all $k \in\{1, \ldots, n\}$

$$
\sum_{i=1}^{k} x_{[i]} \leq \sum_{i=1}^{k} y_{[i]} .
$$

Then we say that $\boldsymbol{x}$ is majorized by $\boldsymbol{y}(\boldsymbol{x} \prec \boldsymbol{y})$.

Definition A.2. A function $\phi: \mathbb{R}_{+}^{n} \rightarrow \mathbb{R}$ is said to be Schur-convex if $\boldsymbol{x} \prec \boldsymbol{y}$ implies $\phi(\boldsymbol{x}) \leq \phi(\boldsymbol{y})$.

Lemma A.3. If $\psi: \mathbb{R}_{+} \rightarrow \mathbb{R}$ is a convex function and

$$
\phi\left(x_{1}, \ldots, x_{n}\right)=\sum_{i=1}^{n} \psi\left(x_{i}\right),
$$

then $\phi$ is Schur-convex.

\section{Proof of Section 3}

Lemma A.4. Given $S$, define $f: \mathbb{R}_{+} \rightarrow \mathbb{N}$ as follows:

$$
f(x)=3 \operatorname{card}(E)+\sum_{e \in E}\left\lceil\frac{\lambda(e)}{2 x}\right\rceil .
$$

Then, for all $n \geq 4 \operatorname{card}(E)$, there exist $\underline{\xi}, \bar{\xi} \in \mathbb{R}_{+}$such that

$$
n \leq f(\xi) \leq n+\operatorname{card}(E), \quad \text { for all } \xi \in[\underline{\xi}, \bar{\xi}) .
$$

Proof. The function $f$ is defined as the sum of a constant and $\operatorname{card}(E)$ terms each one of which is a piecewise constant, weakly decreasing, and right continuous function with jumps of magnitude 1 . Therefore, for all $x_{0}>0$,

$$
0 \leq \lim _{x \rightarrow x_{0}^{-}} f(x)-f\left(x_{0}\right) \leq \operatorname{card}(E) .
$$


Moreover, we have

$$
\begin{aligned}
& \lim _{x \rightarrow \infty} f(x)=3 \operatorname{card}(E)+\operatorname{card}(E)=4 \operatorname{card}(E), \\
& \lim _{x \rightarrow 0^{+}} f(x)=+\infty .
\end{aligned}
$$

Proposition A.5. For a given $S$ there exists $\bar{n} \in \mathbb{N}$ such that for all $n \geq \bar{n}$ there exists $n^{\prime} \in \mathbb{N}$ for which

(a) $n \leq n^{\prime} \leq n+\operatorname{card}(E)$,

(b) the game $\mathscr{H}\left(N_{n^{\prime}}, S, \boldsymbol{\rho}\right)$ admits a Nash equilibrium.

Proof. Let $f$ be defined as in (A.1) and let

$$
\bar{n}=f\left(\frac{\min _{e \in E} \lambda(e)}{10}\right) .
$$

Take $n \geq \bar{n}$. By Lemma A.4 there exists an interval $[\underline{\xi}, \bar{\xi})$ such that, for $\xi \in[\underline{\xi}, \bar{\xi})$ we have $f(\xi)=n^{\prime}$, with $n \leq n^{\prime} \leq n+\operatorname{card}(E)$.

We choose $\xi=\underline{\xi}$ and construct a Nash equilibrium $\boldsymbol{x}^{*}$ of $\mathscr{H}\left(N_{n^{\prime}}, S, \boldsymbol{\rho}\right)$. To do so, we position players on the edges of $S$ as follows.

First case: $e \in E_{L}$. If $e=(v, w)$, with $w \in V_{L}$, then, under $\boldsymbol{x}^{*}$, the number of players on $e$ is

$$
\operatorname{degree}(v)+\left\lceil\frac{\lambda(e)}{2 \xi}\right\rceil+2 .
$$

Out of these players, $\operatorname{degree}(v)$ will be in $v$, and the remaining will be as in Figure 16. Taking into account the number of players on $e$, the length $\lambda(e)$, and the number of intervals of length $\alpha(e) \xi$, we have

$$
\alpha(e)=\frac{\lambda(e)-7 \xi}{\xi\left\lceil\frac{\lambda(e)}{2 \xi}\right]-3 \xi} .
$$

\section{FIGURE 16 ABOUT HERE}

Second case: $e \in E_{I}$. If $e=(v, w)$, with $v, w \in V_{I}$, then, under $\boldsymbol{x}^{*}$, the number of players on $e$ is

$$
\operatorname{degree}(v)+\operatorname{degree}(w)+\left\lceil\frac{\lambda(e)}{2 \xi}\right\rceil+1
$$


Out of these players, $\operatorname{degree}(v)$ will be in $v$, degree $(w)$ will be in $w$, and the remaining will be as in Figure 17. This implies that

$$
\alpha(e)=\frac{\lambda(e)-6 \xi}{\xi\left\lceil\frac{\lambda(e)}{2 \xi}\right\rceil-2 \xi} .
$$

Notice that in this case the construction is still valid if we reverse the roles of $v$ and $w$.

\section{FIGURE 17 ABOUT HERE}

Third case: $e \in E_{L L}$. If $e=(v, w)$, with $v, w \in V_{L}$, then, under $\boldsymbol{x}^{*}$, the number of players on $e$ is

$$
\left\lceil\frac{\lambda(e)}{2 \xi}\right\rceil+3 .
$$

They will be located as in Figure 18. This implies that

$$
\alpha(e)=\frac{\lambda(e)-8 \xi}{\xi\left\lceil\frac{\lambda(e)}{2 \xi}\right\rceil-4 \xi} .
$$

As before, in this case the construction remains valid if we reverse the roles of $v$ and $w$.

\section{FIGURE 18 ABOUT HERE}

The total number of players on $S$ can be easily computed as follows. Given that each vertex $v \in V \backslash V_{L}$ has degree $(v)$ players, there is a total of $2 \operatorname{card}\left(E_{I}\right)+\operatorname{card}\left(E_{L}\right)$ players on the vertices. Moreover, for each $e \in E_{I}$ there are $\lceil\lambda(e) / 2 \xi\rceil+1$ players in the interior of $e$; for each $e \in E_{L}$ there are $\lceil\lambda(e) / 2 \xi\rceil+2$ players in the interior of $e$; for each $e \in E_{L L}$ there are $\lceil\lambda(e) / 2 \xi\rceil+3$ players in the interior of $e$. Hence the total number of players is

$$
\begin{aligned}
& 2 \operatorname{card}\left(E_{I}\right)+\operatorname{card}\left(E_{L}\right)+\operatorname{card}\left(E_{I}\right)+2 \operatorname{card}\left(E_{L}\right)+3 \operatorname{card}\left(E_{L L}\right)+\sum_{e \in E}\left\lceil\frac{\lambda(e)}{2 \xi}\right\rceil \\
& =3 \operatorname{card}(E)+\sum_{e \in E}\left\lceil\frac{\lambda(e)}{2 \xi}\right\rceil=f(\xi)=n^{\prime} .
\end{aligned}
$$

To prove that what we have constructed is a Nash equilibrium, we need to show that for all $e \in E$, we have

$$
1 \leq \alpha(e) \leq 2
$$


Under $\boldsymbol{x}^{*}$, if inequality (A.6) is satisfied, the payoff of each player is between $\xi$ and $2 \xi$. Moreover, if a player deviated on an interval between two other players, then she would obtain a payoff equal to half the length of that interval. If inequality (A.6) is satisfied, then no interval between players is longer than $2 \xi$.

Furthermore, in this construction, all players who share a location with some other player have a payoff equal to $\xi$. This implies that if a player deviates to a location that already has more than one player, then her payoff becomes less than $\xi$. Therefore no player has a profitable deviation.

Claim A.6. For all $e \in E_{L}$, (A.6) holds.

Proof. Given (A.3), it is clear that $\alpha(e) \leq 2$ if and only if

$$
2\left\lceil\frac{\lambda(e)}{2 \xi}\right\rceil-6-\frac{\lambda(e)}{\xi}+7 \geq 0 .
$$

The above inequality holds since

$$
2\left\lceil\frac{\lambda(e)}{2 \xi}\right\rceil-\frac{\lambda(e)}{\xi}+1 \geq 2 \frac{\lambda(e)}{2 \xi}-\frac{\lambda(e)}{\xi}+1=1 \geq 0 .
$$

On the other hand $\alpha(e) \geq 1$ if and only if

$$
\frac{\lambda(e)}{\xi}-7-\left\lceil\frac{\lambda(e)}{2 \xi}\right\rceil+3 \geq 0
$$

To show that the inequality holds consider that

$$
\frac{\lambda(e)}{\xi}-\left\lceil\frac{\lambda(e)}{2 \xi}\right\rceil-4 \geq \frac{\lambda(e)}{\xi}-\frac{\lambda(e)}{2 \xi}-5=\frac{\lambda(e)}{2 \xi}-5 .
$$

Since, by (A.2),

$$
f(\xi)=n^{\prime} \geq \bar{n}=f\left(\frac{\min _{e^{\prime} \in E} \lambda\left(e^{\prime}\right)}{10}\right)
$$

and $f$ is weakly decreasing, we have

$$
\xi \leq \frac{\min _{e^{\prime} \in E} \lambda\left(e^{\prime}\right)}{10} \leq \frac{\lambda(e)}{10} .
$$

Therefore

$$
\frac{\lambda(e)}{2 \xi}-5 \geq 0
$$

Claim A.7. For all $e \in E_{I}$, (A.6) holds. 
Proof. Given (A.4), it is clear that $\alpha(e) \leq 2$ if and only if

$$
2\left\lceil\frac{\lambda(e)}{2 \xi}\right\rceil-4-\frac{\lambda(e)}{\xi}+6 \geq 0 .
$$

The above inequality holds since

$$
2\left\lceil\frac{\lambda(e)}{2 \xi}\right\rceil-\frac{\lambda(e)}{\xi}+2 \geq 2 \frac{\lambda(e)}{2 \xi}-\frac{\lambda(e)}{\xi}+2=2 \geq 0 .
$$

On the other hand $\alpha(e) \geq 1$ if and only if

$$
\frac{\lambda(e)}{\xi}-6-\left\lceil\frac{\lambda(e)}{2 \xi}\right\rceil+2 \geq 0
$$

To show that the inequality holds consider that

$$
\frac{\lambda(e)}{\xi}-\left\lceil\frac{\lambda(e)}{2 \xi}\right\rceil-4 \geq \frac{\lambda(e)}{\xi}-\frac{\lambda(e)}{2 \xi}-5=\frac{\lambda(e)}{2 \xi}-5 .
$$

As mentioned in the proof of Claim A.7, expression (A.2) implies

$$
\frac{\lambda(e)}{2 \xi}-5 \geq 0
$$

Claim A.8. For all $e \in E_{L L}$, (A.6) holds.

Proof. Given (A.5), it is clear that $\alpha(e) \leq 2$ if and only if

$$
2\left\lceil\frac{\lambda(e)}{2 \xi}\right\rceil-8-\frac{\lambda(e)}{\xi}+8 \geq 0 .
$$

The above inequality holds since

$$
2\left\lceil\frac{\lambda(e)}{2 \xi}\right\rceil-\frac{\lambda(e)}{\xi} \geq 2 \frac{\lambda(e)}{2 \xi}-\frac{\lambda(e)}{\xi}=0 .
$$

On the other hand $\alpha(e) \geq 1$ if and only if

$$
\frac{\lambda(e)}{\xi}-8-\left\lceil\frac{\lambda(e)}{2 \xi}\right\rceil+4 \geq 0
$$

To show that the inequality holds consider that

$$
\frac{\lambda(e)}{\xi}-\left\lceil\frac{\lambda(e)}{2 \xi}\right\rceil-4 \geq \frac{\lambda(e)}{\xi}-\frac{\lambda(e)}{2 \xi}-5=\frac{\lambda(e)}{2 \xi}-5 .
$$

But, again, (A.2) implies

$$
\frac{\lambda(e)}{2 \xi}-5 \geq 0
$$


Proof of Theorem 3.1. Proposition A.5 shows that for every

$$
n \geq 3 \operatorname{card}(E)+\sum_{e \in E}\left\lceil\frac{5 \lambda(e)}{\min _{e^{\prime} \in E} \lambda\left(e^{\prime}\right)}\right\rceil
$$

there exists an integer $n \leq n^{\prime} \leq n+\operatorname{card}(E)$ such that the game $\mathscr{H}\left(N_{n^{\prime}}, S, \boldsymbol{\rho}\right)$ admits a Nash equilibrium. Given such an equilibrium, we will construct an equilibrium for a new game with $n^{\prime}-k$ players where $0 \leq k \leq \operatorname{card}(E)$. This can be achieved as follows. We start with the equilibrium of Proposition A.5. For edges $e=(v, w) \in E_{L}$ we can remove one of the two players whose distance from $v$ is $2 \xi$. For edges $e=(v, w) \in E_{I}$ we can remove one of the two players whose distance from $w$ is $2 \xi$. Finally for edges $e=(v, w) \in E_{L L}$ we can remove one of the two players whose distance from $w$ is $3 \xi$. This way we can remove any number $k$ of players with $0 \leq k \leq \operatorname{card}(E)$. We show now that this removal produces a profile that remains an equilibrium in the new game with less players. In fact this removal does not change the size of any interval, it changes the payoff of only $k$ players, who now gain $2 \xi$ rather than $\xi$, therefore, it does not produce any opportunity of profitable deviation for any other player, given that every player gains at least $\xi$.

This proves that, for every $n$ that satisfies (A.7), the game $\mathscr{H}\left(N_{n}, S, \boldsymbol{\rho}\right)$ has an equilibrium.

Definition A.9. A strategy profile $\boldsymbol{x}$ satisfies the vertex property if, for all $v \in V_{I}$, there exists $i \in N_{n}$ such that $x_{i}=v$.

Definition A.10. Let $\boldsymbol{x}$ satisfy the vertex property. Then, for $a, b \in S$, we call $[a, b]$ an $\boldsymbol{x}$-half-interval if either

(i) there exist $e \in E$ and $i \in N_{n}$ such that $b \in e$ is a leaf, $x_{i}=a \in e$, and for no $j \in N$ we have $x_{j} \in(a, b]$, or

(ii) there exist $e \in E, i, \ell \in N$ such that $x_{i}=a \in e, x_{\ell} \in e$, for no $j \in N$ we have $x_{j}$ between $a$ and $x_{\ell}$, and $d([a, b])=d\left(\left[b, x_{\ell}\right]\right)=d\left(\left[a, x_{\ell}\right]\right) / 2$, i.e., $b$ is the middle point between $a$ and $x_{\ell}$.

In both cases the roles of $a$ and $b$ can be interchanged.

In profile $\boldsymbol{x}$, if $m$ players share the same location, then we use the convention that there are $2(m-1)$ zero-length $\boldsymbol{x}$-half intervals between them. If profile $\boldsymbol{x}$ satisfies the vertex property then the whole graph can be covered with $\boldsymbol{x}$-half intervals. We call $H(\boldsymbol{x})$ the class of all $\boldsymbol{x}$-half intervals in $S$.

Lemma A.11. Given a strategy profile $\boldsymbol{x}$, there exists $i \in N$ such that $\rho_{i}(\boldsymbol{x}) \leq L / n$.

Proof. If $\rho_{i}(\boldsymbol{x})>L / n$ for all $i \in N_{n}$, then $\sum_{i=1}^{n} \rho_{i}(\boldsymbol{x})>L$, which is a contradiction, since $\sum_{i=1}^{n} \rho_{i}(\boldsymbol{x})=L$. 
Lemma A.12. If $\boldsymbol{x}^{*}$ is a Nash equilibrium of $\mathscr{H}\left(N_{n}, S, \boldsymbol{\rho}\right)$, then for all $y \in S$ we have $d\left(y, \boldsymbol{x}^{*}\right) \leq 2 L / n$.

Proof. Suppose, by contradiction that there exists $y_{0} \in S$ is such that $d\left(y_{0}, \boldsymbol{x}^{*}\right)>$ $2 L / n$. By Lemma A.11 there exists a player whose payoff is less or equal $L / n$. This player could deviate to $y_{0}$ and then attract at least half the consumers between $y_{0}$ and the closest player, namely she could get a payoff larger than $L / n$, making the deviation profitable.

Lemma A.13. Let $n>\bar{n}$, with $\bar{n}$ defined as in (A.2). Assume that $\boldsymbol{x}^{*}$ is a Nash equilibrium of $\mathscr{H}\left(N_{n}, S, \boldsymbol{\rho}\right)$. Then $\boldsymbol{x}^{*}$ satisfies the vertex property.

Proof. We have

$$
\begin{aligned}
\bar{n} & =f\left(\frac{\min _{e^{\prime} \in E} \lambda\left(e^{\prime}\right)}{10}\right) \\
& =3 \operatorname{card}(E)+\sum_{e \in E}\left\lceil\frac{5 \lambda(e)}{\min _{e^{\prime} \in E} \lambda\left(e^{\prime}\right)}\right] \\
& >3 \operatorname{card}(E)+\sum_{e \in E} \frac{5 \lambda(e)}{\min _{e^{\prime} \in E} \lambda\left(e^{\prime}\right)} \\
& =3 \operatorname{card}(E)+\frac{5 L}{\min _{e^{\prime} \in E} \lambda\left(e^{\prime}\right)} \\
& >\frac{4 L}{\min _{e^{\prime} \in E} \lambda\left(e^{\prime}\right)} .
\end{aligned}
$$

If $n>\bar{n}$, then for any edge $e \in E$ we have $\lambda(e) \geq 4 L / n$ and, therefore, by Lemma A.11, there are at least two players on $e$.

Take $v_{0} \in V_{I}$ and assume, ad absurdum, that no player is in $v_{0}$. Let $i$ the the player whose location $x_{i}^{*}$ is the closest to $v_{0}$ ( $i$ is not necessarily unique). If player $i$ moves towards $v_{0}$ by $\varepsilon<d\left(x_{i}^{*}, v_{0}\right)$, then she loses $\varepsilon / 2$ on the edge where she resides, but she wins (degree $\left.\left(v_{0}\right)-1\right) \varepsilon / 2$ on the other incident edges to $v_{0}$. Therefore moving towards $v_{0}$ is a profitable deviation, which contradicts the assumption that $\boldsymbol{x}^{*}$ is an equilibrium.

Lemma A.14. Let $\boldsymbol{x}$ satisfy the vertex property. Then the number of $\boldsymbol{x}$-half intervals in $S$ is $2 n+2 \operatorname{card}(E)-\operatorname{card}\left(V_{I}\right)-\operatorname{card}(V)$.

Proof. Placing one player on each $v \in V_{I}$ creates $\operatorname{card}(E)$ intervals between two vertices. Every time a new player is placed on some edge, a new interval is created (by splitting an existing interval into two). This is true also if the new player is placed in the same location of an existing player, since this creates two zero-length half intervals. Therefore, once all $n$ players are placed on $S$, there are exactly $\operatorname{card}(E)+n-\operatorname{card}\left(V_{I}\right)$ intervals. Each on them contains two $\boldsymbol{x}$-half intervals, except the ones between a player and a leaf, which contain one half interval. Therefore the number of $\boldsymbol{x}$-half intervals is $2 \operatorname{card}(E)+2 n-\operatorname{card}\left(V_{I}\right)-\operatorname{card}(V)$. 
Lemma A.15. Assume that $\boldsymbol{x}^{*}$ is an equilibrium of $\mathscr{H}\left(N_{n}, S, \boldsymbol{\rho}\right)$ and $[a, b]$ is an $\boldsymbol{x}^{*}$-half interval. Then $\lambda([a, b]) \leq L / n$.

Proof. Assume, ad absurdum, that $\lambda([a, b])>L / n$. By Lemma A.11, there exists $i \in N$ such that $\rho_{i}\left(\boldsymbol{x}^{*}\right) \leq L / n$. Two cases are possible.

Case (i) of Definition A.10. If player $i$ deviates to $[a, b]$ at a distance $\varepsilon$ from $a$, then, for $\varepsilon$ small enough, her payoff becomes $\lambda([a, b])-\varepsilon>L / n$.

Case (ii) of Definition A.10. If player $i$ deviates to $b$, then her payoff becomes $\lambda([a, b])>L / n$.

The existence of profitable deviations contradicts the assumption that $\boldsymbol{x}^{*}$ is a Nash equilibrium.

Lemma A.16. Assume that the conditions of Lemma A.13 are satisfied. Then $\sigma\left(\boldsymbol{x}^{*}\right) \leq L^{2} / 2 n$.

Proof. By Lemma A.13, $\boldsymbol{x}^{*}$ satisfies the vertex property. From the definition of social cost $\sigma$ it follows that

$$
\sigma\left(\boldsymbol{x}^{*}\right)=\sum_{[a, b] \in H\left(\boldsymbol{x}^{*}\right)} \frac{(\lambda([a, b]))^{2}}{2} .
$$

Call $\boldsymbol{\lambda}\left(\boldsymbol{x}^{*}\right)$ the vector of all $\lambda([a, b])$ such that $[a, b] \in H\left(\boldsymbol{x}^{*}\right)$. By Lemma A.15, $\boldsymbol{\lambda}\left(\boldsymbol{x}^{*}\right)$ is dominated in the majorization order by the vector $\left(\begin{array}{llllll}L / n & \ldots & L / n & 0 & \ldots & 0\end{array}\right)$, where the number of positive components is $n$. Since the function $\left(z_{1}, \ldots, z_{m}\right) \mapsto$ $\sum_{i=1}^{m} z_{1}^{2} / 2$ is Schur-convex, we have

$$
\sigma\left(\boldsymbol{x}^{*}\right) \leq \sum_{i=1}^{n} \frac{1}{2}\left(\frac{L}{n}\right)^{2}=\frac{L^{2}}{2 n} .
$$

Lemma A.17. We have

$$
\operatorname{Opt}\left(\mathscr{H}\left(N_{n}, S, \boldsymbol{\rho}\right)\right) \geq \frac{L^{2}}{2\left(2 n+2 \operatorname{card}\left(E_{I}\right)+\operatorname{card}\left(E_{L}\right)\right)} .
$$

Proof. Call $\widetilde{N}_{n}=N_{n} \cup V_{I}$ a fictitious set of players obtained by adding to the original set of players $N_{n}$ one player for each vertex of degree larger than 3 . It is clear that

$$
\operatorname{Opt}\left(\mathscr{H}\left(N_{n}, S, \boldsymbol{\rho}\right)\right) \geq \operatorname{Opt}\left(\widetilde{\mathscr{H}}\left(\widetilde{N}_{n}, S, \boldsymbol{\rho}\right)\right)
$$

where in the game $\widetilde{\mathscr{H}}\left(\widetilde{N}_{n}, S, \boldsymbol{\rho}\right)$ each player in $V_{I}$ has only one available strategy, that is, to choose the location corresponding to her own identity. 
Call $\tilde{\boldsymbol{x}}$ the optimal profile of $\widetilde{\mathscr{H}}\left(\widetilde{N}_{n}, S, \boldsymbol{\rho}\right)$. Applying the argument used in Lemma A.14 to this new game, we can show that the number of $\tilde{\boldsymbol{x}}$-half intervals is

$$
\begin{aligned}
M & :=2 n+2 \operatorname{card}(E)-\operatorname{card}(V)+\operatorname{card}\left(V_{I}\right) \\
& =2 n+2 \operatorname{card}(E)-\operatorname{card}\left(V_{L}\right) \\
& =2 n+2 \operatorname{card}(E)-\operatorname{card}\left(E_{L}\right)-2 \operatorname{card}\left(E_{L L}\right) \\
& =2 n+2 \operatorname{card}\left(E_{I}\right)+\operatorname{card}\left(E_{L}\right) .
\end{aligned}
$$

It is clear that $\boldsymbol{\lambda}(\tilde{\boldsymbol{x}})$ dominates the vector $(L / M \quad \ldots L / M)$. Since

$$
\sum_{i=1}^{M} \frac{1}{2}\left(\frac{L}{M}\right)^{2}=\frac{L^{2}}{2 M}
$$

we have

$$
\operatorname{Opt}\left(\mathscr{H}\left(N_{n}, S, \boldsymbol{\rho}\right)\right) \geq \operatorname{Opt}\left(\widetilde{\mathscr{H}}\left(\widetilde{N}_{n}, S, \boldsymbol{\rho}\right)\right) \geq \frac{L^{2}}{2 M}
$$

Proof of Theorem 3.2(a). The results follows from the bounds in Lemma A.16 and in Lemma A.17.

Claim A.18. Consider the equilibrium $\boldsymbol{x}^{*}$ constructed in the proof of Proposition A.5. Then

$$
\begin{aligned}
& \sigma\left(\boldsymbol{x}^{*}\right)=\sum_{e \in E_{L}}\left(7 \frac{\xi^{2}}{2}+\left(\left\lceil\frac{\lambda(e)}{2 \xi}\right\rceil-3\right) \frac{\alpha(e)^{2} \xi^{2}}{4}\right) \\
& +\sum_{e \in E_{I}}\left(6 \frac{\xi^{2}}{2}+\left(\left\lceil\frac{\lambda(e)}{2 \xi}\right\rceil-2\right) \frac{\alpha(e)^{2} \xi^{2}}{4}\right)+\sum_{e \in E_{L L}}\left(8 \frac{\xi^{2}}{2}+\left(\left\lceil\frac{\lambda(e)}{2 \xi}\right\rceil-4\right) \frac{\alpha(e)^{2} \xi^{2}}{4}\right) .
\end{aligned}
$$

Proof. Each edge $e \in E_{L}$ contains 7 half intervals of length $\xi$ and $\left\lceil\frac{\lambda(e)}{2 \xi}\right\rceil-3$ intervals of length $\alpha(e) \xi$. The cost of edge $e$ is then

$$
7 \frac{\xi^{2}}{2}+\left(\left\lceil\frac{\lambda(e)}{2 \xi}\right\rceil-3\right) \frac{\alpha(e)^{2} \xi^{2}}{4} .
$$

Each edge $e \in E_{I}$ contains 6 half intervals of length $\xi$ and $\left\lceil\frac{\lambda(e)}{2 \xi}\right\rceil-2$ intervals of length $\alpha(e) \xi$. The cost of edge $e$ is then

$$
6 \frac{\xi^{2}}{2}+\left(\left\lceil\frac{\lambda(e)}{2 \xi}\right\rceil-2\right) \frac{\alpha(e)^{2} \xi^{2}}{4} .
$$

Each edge $e \in E_{L L}$ contains 8 half intervals of length $\xi$ and $\left\lceil\frac{\lambda(e)}{2 \xi}\right\rceil-4$ intervals of length $\alpha(e) \xi$. The cost of edge $e$ is then

$$
8 \frac{\xi^{2}}{2}+\left(\left\lceil\frac{\lambda(e)}{2 \xi}\right\rceil-4\right) \frac{\alpha(e)^{2} \xi^{2}}{4} .
$$




\section{Claim A.19.}

$$
\frac{L}{2 n-4 \operatorname{card}(E)} \leq \xi \leq \frac{L}{2 n-6 \operatorname{card}(E)} .
$$

Proof. By definition, $\xi$ is such that $f(\xi)=n^{\prime}$ with $n \leq n^{\prime} \leq n+\operatorname{card}(E)$, where $f$ is defined as in (A.1). Therefore

$$
n \leq 3 \operatorname{card}(E)+\sum_{e \in E}\left\lceil\frac{\lambda(e)}{2 \xi}\right\rceil \leq n+\operatorname{card}(E),
$$

which implies

$$
\frac{L}{2 n-4 \operatorname{card}(E)} \leq \xi \leq \frac{L}{2 n-6 \operatorname{card}(E)} .
$$

Claim A.20. For all $e \in E$ we have $\lim _{n \rightarrow \infty} \alpha(e)=2$.

Proof. Claim A.19 implies

$$
\xi \sim_{n \rightarrow \infty} \frac{L}{2 n}
$$

Therefore, if $e \in E_{L}$, then

$$
\alpha(e)=\frac{\lambda(e)-7 \xi}{\xi\left\lceil\frac{\lambda(e)}{2 \xi}\right\rceil-3 \xi} \sim_{n \rightarrow \infty} 2 ;
$$

if $e \in E_{L}$, then

$$
\alpha(e)=\frac{\lambda(e)-6 \xi}{\xi\left\lceil\frac{\lambda(e)}{2 \xi}\right\rceil-2 \xi} \sim_{n \rightarrow \infty} 2
$$

if $e \in E_{L L}$, then

$$
\alpha(e)=\frac{\lambda(e)-8 \xi}{\xi\left\lceil\frac{\lambda(e)}{2 \xi}\right\rceil-4 \xi} \sim_{n \rightarrow \infty} 2 .
$$

Claim A.21.

$$
\sigma\left(\boldsymbol{x}^{*}\right) \sim_{n \rightarrow \infty} \frac{L^{2}}{4 n} .
$$

Proof. Using Claims A.18 and A.20, we have

$$
\sigma\left(\boldsymbol{x}^{*}\right) \sim_{\xi \rightarrow 0} \sum_{e \in E_{L}}\left(\frac{\xi^{2}}{2}+\frac{\lambda(e) \xi}{2}\right)+\sum_{e \in E_{I}}\left(\xi^{2}+\frac{\lambda(e) \xi}{2}\right)+\sum_{e \in E_{L L}}\left(2 \xi^{2}+\frac{\lambda(e) \xi}{2}\right) .
$$

Hence

$$
\sigma\left(\boldsymbol{x}^{*}\right) \sim_{\xi \rightarrow 0} \sum_{e \in E} \frac{\lambda(e) \xi}{2},
$$

that is, thanks to (A.8),

$$
\sigma\left(\boldsymbol{x}^{*}\right) \sim_{n \rightarrow \infty} \frac{L^{2}}{2 n}
$$


Proof of Theorem 3.2(b). By Lemma A.17 we have

$$
\operatorname{Opt}\left(\mathscr{H}\left(N_{n}, S, \boldsymbol{\rho}\right)\right) \geq \frac{L^{2}}{4 n+4 \operatorname{card}\left(E_{I}\right)+2 \operatorname{card}\left(E_{L}\right)} .
$$

Therefore

$$
\operatorname{IPoS}\left(\mathscr{H}\left(N_{n}, S, \boldsymbol{\rho}\right)\right) \leq \frac{\sigma\left(\boldsymbol{x}^{*}\right)}{\frac{L^{2}}{4 n+4 \operatorname{card}\left(E_{I}\right)+2 \operatorname{card}\left(E_{L}\right)}}
$$

Since

$$
\frac{L^{2}}{\left.4 n+4 \operatorname{card}\left(E_{I}\right)+2 \operatorname{card}\left(E_{L}\right)\right)} \sim_{n \rightarrow \infty} \frac{L^{2}}{4 n}
$$

and $\operatorname{IPoS}\left(\mathscr{H}\left(N_{n}, S, \boldsymbol{\rho}\right)\right) \geq 1$, using Claim A.21, we obtain

$$
\lim _{n \rightarrow \infty} \operatorname{IPoS}\left(\mathscr{H}\left(N_{n}, S, \boldsymbol{\rho}\right)\right)=1
$$

\section{Proofs of Subsection 4.1}

The set action for the players is now a circle $C$ with radius $r$. Without loss of generality we can suppose that $r=1$. We fix an origin 0 and identify the circle $C$ with $\mathbb{R} / 2 \pi$. We will always consider the representation of the points in $[0,2 \pi)$.

Given a strategy profile $\boldsymbol{x} \in C^{n}$, for each player $i \in N_{n}$, we define two domains $\overleftarrow{p}_{i}(\boldsymbol{x})$ and $\vec{p}_{i}(\boldsymbol{x})$ as follows. If we order the players $i_{1} \prec \cdots \prec i_{n}$ in terms of their actions $0 \leq x_{i_{1}} \leq \cdots \leq x_{i_{n}}<2 \pi$ (solve the ties arbitrarily). Make the relation $\prec$ cyclic by assuming $i_{n} \prec i_{1}$. For $\ell \in N_{n}$ define

$$
\begin{aligned}
& \overleftarrow{p}_{i_{\ell}}(\boldsymbol{x})=\frac{1}{2} d\left(x_{i_{\ell-1}}, x_{i_{\ell}}\right) \\
& \vec{p}_{i_{\ell}}(\boldsymbol{x})=\frac{1}{2} d\left(x_{i_{\ell}}, x_{i_{\ell+1}}\right)
\end{aligned}
$$

where $i_{0}:=i_{n}$ and $i_{n+1}:=i_{1}$.

Claim A.22. For $i \in N_{n}$

$$
\rho_{i}(\boldsymbol{x})=\frac{1}{\operatorname{card}\left\{\ell: x_{\ell}=x_{i}\right\}} \sum_{\ell: x_{\ell}=x_{i}}\left(\overleftarrow{p}_{\ell}(\boldsymbol{x})+\vec{p}_{\ell}(\boldsymbol{x})\right)
$$

Proof. This is immediate from the definition of $\rho, \vec{p}_{\ell}(\boldsymbol{x})$ and $\overleftarrow{p}_{\ell}(\boldsymbol{x})$

The proof of Proposition 4.1 is based on the following lemma.

Lemma A.23. If the profile $\boldsymbol{x}^{*}$ is an equilibrium of the Hotelling game $\mathscr{H}\left(N_{n}, C, \boldsymbol{\rho}\right)$ then the following conditions are satisfied: 
(a) For every $y \in[0,1]$

$$
\operatorname{card}\left\{i: x_{i}^{*}=y\right\} \leq 2 .
$$

(b) There exist $\xi>0$ such that for all $y \in C$, if

$$
\operatorname{card}\left\{i: x_{i}^{*}=y\right\}=2 \text {, }
$$

then

$$
\overleftarrow{p}_{i}\left(\boldsymbol{x}^{*}\right)=0 \text { and } \vec{p}_{i}\left(\boldsymbol{x}^{*}\right)=\xi
$$

or

$$
\vec{p}_{i}\left(\boldsymbol{x}^{*}\right)=0 \text { and } \overleftarrow{p}_{i}\left(\boldsymbol{x}^{*}\right)=\xi
$$

(c) For every $i \in N_{n}$,

$$
\max _{i_{j} \in N_{n}} d\left(x_{i_{j}}^{*}, x_{i_{j}+1}^{*}\right) \leq 2\left(\vec{p}_{i}\left(\boldsymbol{x}^{*}\right)+\overleftarrow{p}_{i}\left(\boldsymbol{x}^{*}\right)\right)
$$

Proof. (a) Suppose, ad absurdum, that there exist a value $y \in[0,1]$ such that $\{i \in$ $\left.N_{n}: x_{i}^{*}=y\right\}=\left\{i_{1}, \ldots, i_{\ell}\right\}$ with $\ell>2$. Then for some $\alpha, \beta>0$

$$
\begin{aligned}
& \overleftarrow{p}_{i_{1}}\left(\boldsymbol{x}^{*}\right)=\alpha, \quad \vec{p}_{i_{1}}\left(\boldsymbol{x}^{*}\right)=0 \\
& \text { for all } j \in\{2, \ldots, \ell-1\}, \quad \overleftarrow{p}_{i_{j}}\left(\boldsymbol{x}^{*}\right)=\vec{p}_{i_{j}}\left(\boldsymbol{x}^{*}\right)=0 \\
& \overleftarrow{p}_{i_{\ell}}\left(\boldsymbol{x}^{*}\right)=0, \quad \vec{p}_{i_{\ell}}\left(\boldsymbol{x}^{*}\right)=\beta
\end{aligned}
$$

This implies

$$
\sum_{i=1}^{\ell} \rho_{i}\left(\boldsymbol{x}^{*}\right)=\alpha+\beta
$$

therefore

$$
\rho_{j}\left(\boldsymbol{x}^{*}\right)=\frac{\alpha+\beta}{\ell}<\frac{\alpha+\beta}{2}<\max (\alpha, \beta) \text { for all } j \in\{1, \ell\} .
$$

For $\varepsilon$ small enough any player $j \in\left\{i_{1}, \ldots i_{\ell}\right\}$ would get $\alpha-\varepsilon$ by playing $y-\varepsilon$ or $\beta-\varepsilon$ by playing $y+\varepsilon$, so this would be a profitable deviation.

(b) Let $y \in[0,1]$ be such that $x_{i_{1}}^{*}=x_{i_{2}}^{*}=y$ and $\operatorname{card}\left\{i: x_{i}=y\right\}=2$. Then for some $\alpha, \beta>0$

$$
\begin{aligned}
& \vec{p}_{i_{1}}\left(\boldsymbol{x}^{*}\right)=\overleftarrow{p}_{i_{2}}\left(\boldsymbol{x}^{*}\right)=0 \\
& \overleftarrow{p}_{i_{1}}\left(\boldsymbol{x}^{*}\right)=\alpha \\
& \vec{p}_{i_{2}}\left(\boldsymbol{x}^{*}\right)=\beta
\end{aligned}
$$

We know that

$$
\rho_{i_{1}}\left(\boldsymbol{x}^{*}\right)=\rho_{i_{2}}\left(\boldsymbol{x}^{*}\right)=\frac{\alpha+\beta}{2} \leq \max (\alpha, \beta)
$$


with a strict inequality if $\alpha \neq \beta$. If this were the case, then there would be a profitable deviation since for some $\varepsilon>0$ both $i_{1}$ and $i_{2}$ could win $\alpha-\varepsilon$ by playing $y-\varepsilon$ and $\beta-\varepsilon$ by playing $y+\varepsilon$. Denote $\xi:=\alpha=\beta$ and consider $y^{\prime} \neq y$ such that $x_{i_{3}}^{*}=x_{i_{4}}^{*}=y^{\prime}$ and $\operatorname{card}\left\{i: x_{i}=y^{\prime}\right\}=2$. Then

$$
\rho_{i_{3}}\left(\boldsymbol{x}^{*}\right)=\rho_{i_{4}}\left(\boldsymbol{x}^{*}\right)=\frac{\overleftarrow{p}_{i_{3}}\left(\boldsymbol{x}^{*}\right)+\vec{p}_{i_{4}}\left(\boldsymbol{x}^{*}\right)}{2}=: \xi^{\prime}
$$

If $\xi<\xi^{\prime}$ player 1 (or 2) can deviate to $y^{\prime}+\varepsilon$ and win $\xi^{\prime}+\varepsilon$. If $\xi>\xi^{\prime}$ player 3 (or 4 ) can deviate to $y+\varepsilon$ and win $\xi+\varepsilon$. Therefore we must have $\xi=\xi^{\prime}$.

(c) If (A.9) does not hold, then player $i$ has an incentive to deviate anywhere in the arc between $x_{i_{j}}^{*}$ and $x_{i_{j}+1}^{*}$.

Proof of Proposition 4.1. If a profile $\boldsymbol{x}^{*}$ satisfies conditions (a), (b), (c) of Lemma A.23, then no deviation to an interval between two players can be profitable. Suppose, ad absurdum, that player $i$ has an incentive to deviate in a location $y$ occupied by player $j$ (and by nobody else, since we proved that, in equilibrium, we never have more than two players in the same location). This implies that the payoffs of players $i$ and $j$ before the deviation satisfy $\rho_{j}\left(\boldsymbol{x}^{*}\right)>2 \rho_{i}\left(\boldsymbol{x}^{*}\right)$, that is,

$$
\begin{aligned}
\rho_{j}\left(\boldsymbol{x}^{*}\right) & =\overleftarrow{p}_{j}\left(\boldsymbol{x}^{*}\right)+\vec{p}_{j}(\boldsymbol{x}) \\
& =\frac{d\left(x_{j-1}^{*}, x_{j}^{*}\right)+d\left(x_{j}^{*}, x_{j+1}^{*}\right)}{2} \\
& >2 \rho_{i}\left(\boldsymbol{x}^{*}\right)
\end{aligned}
$$

which implies either $d\left(x_{j-1}^{*}, x_{j}^{*}\right)>2 \rho_{i}\left(\boldsymbol{x}^{*}\right)$ or $d\left(x_{j}^{*}, x_{j+1}^{*}\right)>2 \rho_{i}\left(\boldsymbol{x}^{*}\right)$. But this contradicts (A.9). So $\boldsymbol{x}^{*}$ is an equilibrium. Lemma A.25 below shows that for every $n \in \mathbb{N}$ there exist strategy profiles that satisfy conditions (a), (b), (c) of Lemma A.23. Given that, for each player, the set of equilibria is determined by a finite number of linear inequalities, we have that this set is a polytope.

Lemma A.24. For every $\boldsymbol{x} \in C$ we have

$$
\sigma(\boldsymbol{x})=\sum_{i=1}^{n} \frac{\left(x_{i+1}-x_{i}\right)^{2}}{4}=\frac{\sum_{i=1}^{n}\left[\left(\overleftarrow{p}_{i}(\boldsymbol{x})\right)^{2}+\left(\vec{p}_{i}(\boldsymbol{x})\right)^{2}\right]}{2}
$$

where $x_{n+1}:=x_{1}$.

Proof. This is immediate from the definition of $\sigma, \vec{p}_{i}(\boldsymbol{x})$ and $\overleftarrow{p}_{i}(\boldsymbol{x})$

Lemma A.25. Consider the game $\mathscr{H}\left(N_{n}, C, \boldsymbol{\rho}\right)$. Call $\tilde{\boldsymbol{x}}$ the action profile such that

$$
\tilde{x}_{i}=\frac{i}{n} 2 \pi \text {. }
$$


For $n$ even call $\tilde{\tilde{\boldsymbol{x}}}$ the action profile such that

$$
\tilde{\tilde{x}}_{2 i-1}=\tilde{\tilde{x}}_{2 i}=\frac{2 i}{n} 2 \pi, \quad i \in\{1, \ldots, n / 2\} .
$$

For $n$ odd call $\tilde{\tilde{\boldsymbol{x}}}^{\perp}$ the action profile such that

$$
\begin{aligned}
& \tilde{\tilde{x}}_{2 i-1}=\tilde{\tilde{x}}_{2 i}=\frac{2 i}{n} 2 \pi, \quad i \in\{1, \ldots,\lfloor n / 2\rfloor\} \\
& x_{n}=2 \pi
\end{aligned}
$$

(a) The action profiles $\tilde{\boldsymbol{x}}, \tilde{\tilde{\boldsymbol{x}}}$, and $\tilde{\tilde{\boldsymbol{x}}}^{\perp}$ are equilibria in $\mathscr{H}\left(N_{n}, C, \boldsymbol{\rho}\right)$.

(b) For all positive $n$

$$
\begin{aligned}
& \tilde{\boldsymbol{x}} \in \arg \min _{\boldsymbol{x} \in \mathscr{E}(\mathscr{H})} \sigma(\boldsymbol{x}), \\
& \tilde{\boldsymbol{x}} \in \arg \min _{\boldsymbol{x} \in C^{n}} \sigma(\boldsymbol{x}),
\end{aligned}
$$

and

$$
\text { Opt } \mathscr{H}\left(N_{n}, C, \boldsymbol{\rho}\right)=\operatorname{BEq} \mathscr{H}\left(N_{n}, C, \boldsymbol{\rho}\right)=\sigma(\tilde{\boldsymbol{x}})=\frac{\pi^{2}}{n}
$$

(c) For $n$ even

$$
\tilde{\tilde{\boldsymbol{x}}} \in \arg \max _{\boldsymbol{x} \in \mathscr{E}(\mathscr{H})} \sigma(\boldsymbol{x})
$$

and

$$
\text { WEq } \mathscr{H}\left(N_{n}, C, \boldsymbol{\rho}\right)=\sigma(\tilde{\tilde{\boldsymbol{x}}})=\frac{2 \pi^{2}}{n} \text {. }
$$

(d) For $n$ odd

$$
\tilde{\tilde{\boldsymbol{x}}}^{\perp} \in \arg \max _{\boldsymbol{x} \in \mathscr{E}(\mathscr{H})} \sigma(\boldsymbol{x})
$$

and

$$
\text { WEq } \mathscr{H}\left(N_{n}, C, \boldsymbol{\rho}\right)=\sigma\left(\tilde{\tilde{\boldsymbol{x}}}^{\perp}\right)=\frac{2 \pi^{2}}{n+1} .
$$

Proof. (a) These profiles satisfy the conditions of Lemma A.23.

(b) Define

$$
\boldsymbol{p}(\boldsymbol{x}):=\left(\overleftarrow{p}_{1}(\boldsymbol{x}), \vec{p}_{1}(\boldsymbol{x}), \ldots, \overleftarrow{p}_{n}(\boldsymbol{x}), \vec{p}_{n}(\boldsymbol{x})\right) \in \mathbb{R}^{2 n}
$$

Since, for all $i \in N_{n}$,

$$
\overleftarrow{p}_{i}(\tilde{\boldsymbol{x}})=\vec{p}_{i}(\tilde{\boldsymbol{x}})=\frac{2 \pi}{2 n}
$$

we have

$$
\boldsymbol{p}(\tilde{\boldsymbol{x}})=\left(\frac{\pi}{n}, \ldots, \frac{\pi}{n}\right) \in \mathbb{R}^{2 n}
$$


Then for all $\boldsymbol{x} \in C^{n}$ we have $\boldsymbol{p}(\tilde{\boldsymbol{x}}) \prec \boldsymbol{p}(\boldsymbol{x})$. Hence, using Lemma A.27 and Schurconvexity of the function

$$
\boldsymbol{p}(\boldsymbol{x}) \mapsto \frac{\sum_{i=1}^{n}\left[\left(\overleftarrow{p}_{i}(\boldsymbol{x})\right)^{2}+\left(\vec{p}_{i}(\boldsymbol{x})\right)^{2}\right]}{2},
$$

for every $\boldsymbol{x} \in C^{n}$ we have

$$
\sigma(\tilde{\boldsymbol{x}}) \leq \sigma(\boldsymbol{x})
$$

which proves (A.11).

Expression (A.10) follows from the fact that $\tilde{\boldsymbol{x}}$ is an equilibrium.

To prove (A.12) consider that the cost generated by a half domain $p_{i}(\overline{\boldsymbol{x}})$ is $\int_{0}^{\pi / n} x \mathrm{~d} \lambda(x)=\pi^{2} / 2 n^{2}$ and there are $2 n$ such half domains (See Figure 2).

(c) For $i$ even

$$
\vec{p}_{i}(\tilde{\tilde{\boldsymbol{x}}})=\frac{2 \pi}{n}, \quad \overleftarrow{p}_{i}(\tilde{\tilde{\boldsymbol{x}}})=0
$$

Lemma A.23(c) implies

$$
\vec{p}_{i}(\boldsymbol{x})+\overleftarrow{p}_{i}(\boldsymbol{x}) \leq \frac{2 \pi}{n} \quad \text { for all } \boldsymbol{x} \in \mathscr{E}(\mathscr{H})
$$

Therefore,

$$
\boldsymbol{p}(\boldsymbol{x}) \prec \boldsymbol{p}(\tilde{\tilde{\boldsymbol{x}}}) \quad \text { for all } \boldsymbol{x} \in \mathscr{E}(\mathscr{H}) .
$$

Again Lemma A.24 and the usual Schur-convexity argument provide (A.13).

To prove (A.14) consider that the cost generated by $n$ of the half domains $p_{i}(\hat{\boldsymbol{x}})$ is $\int_{0}^{2 \pi / n} x \mathrm{~d} \lambda(x)=2 \pi^{2} / n^{2}$ and the cost generated by the remaining $n$ half domains is 0 (See Figure 3).

(d) To prove the A.16 consider that the cost generated by $n+1$ of the half domains $p_{i}(\hat{\boldsymbol{x}})$ is $\int_{0}^{2 \pi /(n+1)} x \mathrm{~d} \lambda(x)=2 \pi^{2} /(n+1)^{2}$ and the cost generated by the remaining $n$ half domains is 0 (See Figure 4).

Proof of Proposition 4.2. (a) This follows from Lemma A.25(b), (c), and (d).

(b) This is just Lemma A.25(b).

\section{Proofs of Subsection 4.2}

Given a strategy profile $\boldsymbol{x}$, for each player $i \in N_{n}$, we define two domains $\overleftarrow{p}_{i}(\boldsymbol{x})$ and $\vec{p}_{i}(\boldsymbol{x})$ as follows. Order the players $i_{1} \prec \cdots \prec i_{n}$ in terms of their actions $0 \leq x_{i_{1}}, \leq \cdots \leq x_{i_{n}} \leq 1$ (solve the ties arbitrarily). For $\ell \in\{2, \ldots, n-1\}$

$$
\begin{aligned}
& \overleftarrow{p}_{i_{\ell}}(\boldsymbol{x})=\frac{1}{2} d\left(x_{i_{\ell-1}}, x_{i_{\ell}}\right) \\
& \vec{p}_{i_{\ell}}(\boldsymbol{x})=\frac{1}{2} d\left(x_{i_{\ell}}, x_{i_{\ell+1}}\right) \\
& \overleftarrow{p}_{i_{1}}(\boldsymbol{x})=d\left(0, x_{i_{1}}\right) \\
& \vec{p}_{i_{n}}(\boldsymbol{x})=d\left(x_{i_{n}}, 1\right)
\end{aligned}
$$


The following lemma will be the building block of most of all our results in the sequel.

Lemma A.26. Let $\boldsymbol{x}^{*}$ be a Nash equilibrium of $\mathscr{H}\left(N_{n},[0,1], \boldsymbol{\rho}\right)$. Then

(a) For $i \in N_{n}$

$$
\rho_{i}(\boldsymbol{x})=\frac{1}{\operatorname{card}\left\{\ell: x_{\ell}=x_{i}\right\}} \sum_{\ell: x_{\ell}=x_{i}}\left(\overleftarrow{p}_{\ell}(\boldsymbol{x})+\vec{p}_{\ell}(\boldsymbol{x})\right)
$$

(b) For every $y \in[0,1]$

$$
\operatorname{card}\left\{i: x_{i}^{*}=y\right\} \leq 2 .
$$

(c) There exist $\xi>0$ such that for all $y \in[0,1]$, if

$$
\operatorname{card}\left\{i: x_{i}^{*}=y\right\}=2 \text {, }
$$

then

$$
\overleftarrow{p}_{i}\left(\boldsymbol{x}^{*}\right)=0 \text { and } \vec{p}_{i}\left(\boldsymbol{x}^{*}\right)=\xi
$$

or

$$
\vec{p}_{i}\left(\boldsymbol{x}^{*}\right)=0 \text { and } \overleftarrow{p}_{i}\left(\boldsymbol{x}^{*}\right)=\xi
$$

(d) Players on the extremes are coupled, i.e., $x_{i_{1}}=x_{i_{2}}$ and $x_{i_{n-1}}=x_{i_{n}}$.

Proof. (a) Follow immediatly from the definition of payoffs.

(b) Suppose, ad absurdum, that there exist an equilibrium $\boldsymbol{x}^{*}$ and a value $y \in[0,1]$ such that $\left\{i: x_{i}^{*}=y\right\}=\left\{i_{1}, \ldots, i_{\ell}\right\}$ with $\ell>2$. Then for some $\alpha, \beta>0$

$$
\begin{aligned}
& \overleftarrow{p}_{i_{1}}\left(\boldsymbol{x}^{*}\right)=\alpha, \quad \vec{p}_{i_{1}}\left(\boldsymbol{x}^{*}\right)=0 \\
& \text { for all } j \in\{2, \ldots, \ell-1\}, \quad \overleftarrow{p}_{i_{j}}\left(\boldsymbol{x}^{*}\right)=\vec{p}_{i_{j}}\left(\boldsymbol{x}^{*}\right)=0, \\
& \overleftarrow{p}_{i_{\ell}}\left(\boldsymbol{x}^{*}\right)=0, \quad \vec{p}_{i_{\ell}}\left(\boldsymbol{x}^{*}\right)=\beta
\end{aligned}
$$

This implies

$$
\sum_{i=1}^{\ell} \rho_{i}\left(\boldsymbol{x}^{*}\right)=\alpha+\beta
$$

therefore

$$
\rho_{j}\left(\boldsymbol{x}^{*}\right)=\frac{\alpha+\beta}{\ell}<\frac{\alpha+\beta}{2}<\max (\alpha, \beta) \quad \text { for all } j \in\{1, \ell\} .
$$

For $\varepsilon$ small enough any player $j \in\left\{i_{1}, \ldots i_{\ell}\right\}$ would get $\alpha-\varepsilon$ by playing $y-\varepsilon$ or $\beta-\varepsilon$ by playing $y+\varepsilon$, so this would be a profitable deviation. 
(c) Let $y \in[0,1]$ be such that $x_{i_{1}}^{*}=x_{i_{2}}^{*}=y$ and $\operatorname{card}\left\{i: x_{i}=y\right\}=2$. Then for some $\alpha, \beta>0$

$$
\begin{aligned}
& \vec{p}_{i_{1}}\left(\boldsymbol{x}^{*}\right)=\overleftarrow{p}_{i_{2}}\left(\boldsymbol{x}^{*}\right)=0 \\
& \overleftarrow{p}_{i_{1}}\left(\boldsymbol{x}^{*}\right)=\alpha \\
& \vec{p}_{i_{2}}\left(\boldsymbol{x}^{*}\right)=\beta
\end{aligned}
$$

We know that

$$
\rho_{i_{1}}\left(\boldsymbol{x}^{*}\right)=\rho_{i_{2}}\left(\boldsymbol{x}^{*}\right)=\frac{\alpha+\beta}{2} \leq \max (\alpha, \beta),
$$

with a strict inequality if $\alpha \neq \beta$. If this were the case, then there would be a profitable deviation since for some $\varepsilon>0$ both $i_{1}$ and $i_{2}$ could win $\alpha-\varepsilon$ by playing $y-\varepsilon$ and $\beta-\varepsilon$ by playing $y+\varepsilon$. Denote $\xi:=\alpha=\beta$ and consider $y^{\prime} \neq y$ such that $x_{i_{3}}^{*}=x_{i_{4}}^{*}=y^{\prime}$ and $\operatorname{card}\left\{i: x_{i}=y^{\prime}\right\}=2$. Then

$$
\rho_{i_{3}}\left(\boldsymbol{x}^{*}\right)=\rho_{i_{4}}\left(\boldsymbol{x}^{*}\right)=\frac{\overleftarrow{p}_{i_{3}}\left(\boldsymbol{x}^{*}\right)+\vec{p}_{i_{4}}\left(\boldsymbol{x}^{*}\right)}{2}=: \xi^{\prime}
$$

If $\xi<\xi^{\prime}$ player 1 (or 2) can deviate to $y^{\prime}+\varepsilon$ and win $\xi^{\prime}+\varepsilon$. If $\xi>\xi^{\prime}$ player 3 (or 4 ) can deviate to $y+\varepsilon$ and win $\xi+\varepsilon$. Therefore we must have $\xi=\xi^{\prime}$.

(d) Suppose that $x_{i_{1}}<x_{i_{2}}$. Since

$$
\rho_{i_{1}}=\overleftarrow{p}_{i_{1}}\left(\boldsymbol{x}^{*}\right)+\vec{p}_{i_{1}}\left(\boldsymbol{x}^{*}\right)=d\left(0, x_{i_{1}}^{*}\right)+\frac{1}{2} d\left(x_{i_{1}}^{*}, x_{i_{2}}^{*}\right)
$$

player $i_{1}$ would have a profitable deviation by playing $\left(x_{i_{1}}^{*}+x_{i_{2}}^{*}\right) / 2$. The same argument holds for $i_{n-1}$ and $i_{n}$.

For the sake of completeness we give a full proof of Proposition 4.3, even if some of the cases are known (see, e.g., Huck, Müller, and Vriend, 2002, Brenner, 2005, Pálvölgyi, 2011).

Proof of Proposition 4.3. (a) Consider the case $n=2$. The strategy profile $\left(\frac{1}{2}, \frac{1}{2}\right)$ is a Nash equilibrium. The payoff for each player is $1 / 2$, whereas any deviation would provide a lower payoff. It is unique, since for any profile $\left(x_{1}, x_{2}\right) \neq(1 / 2,1 / 2)$ both players would rather deviate to $\left(x_{1}+x_{2}\right) / 2$ if $x_{1}$ and $x_{2}$ are on different sides of $(1 / 2,1 / 2)$, or to $1 / 2$ if they are on the same side (see Figure 5).

Consider now the case $n=4$. We show that the profile $(1 / 4,1 / 4,3 / 4,3 / 4)$ is the unique Nash equilibrium. In fact, by Lemma A.26(d), for some permutation $i_{1}, i_{2}, i_{3}, i_{4}$, we must have $x_{i_{1}}=x_{i_{2}}$ and $x_{i_{3}}=x_{i_{4}}$. Moreover, by Lemma A.26(c), we have

$$
x_{i_{1}}^{*}=\xi=1-x_{i_{4}}^{*}, \text { and } x_{i_{4}}^{*}-x_{i_{1}}^{*}=2 \xi .
$$

Hence $\xi=1 / 4$ (see Figure 6). 
Finally, consider the case $n=5$. We show that the profile $(1 / 6,1 / 6,1 / 2,5 / 6,5 / 6)$ is the unique Nash equilibrium. By Lemma A.26(d), for some permutation $i_{1}, i_{2}, i_{3}, i_{4}, i_{5}$, we have $x_{i_{1}}^{*}=x_{i_{2}}^{*}$ and $x_{i_{4}}^{*}=x_{i_{5}}^{*}$. Moreover, by Lemma A.26(c), we have

$$
x_{i_{1}}^{*}=\xi=1-x_{i_{5}}^{*}, \text { and } x_{i_{4}}^{*}-x_{i_{3}}^{*}=x_{i_{3}}^{*}-x_{i_{2}}^{*}=2 \xi .
$$

Hence $\xi=1 / 6$ (see Figure 7).

(b) By Lemma A.26(b) in equilibrium we cannot have $x_{1}^{*}=x_{2}^{*}=x_{3}^{*}$. For any configuration different from this, one of the players whose action is not the middle one, if she is alone in taking that action, will want to deviate closer to her opponents.

(c) We start with necessary conditions for $\boldsymbol{x}^{*}$ to be a pure Nash equilibrium. By Lemma A.26(c) and (d)

(i) $x_{i_{1}}^{*}=x_{i_{2}}^{*}=1-x_{i_{n-1}}^{*}=1-x_{i_{n}}^{*}=\xi$,

(ii) $x_{i_{3}}^{*}-x_{i_{2}}^{*}=x_{i_{n-1}}^{*}-x_{i_{n-2}}^{*}=2 \xi$,

(iii) $x_{i_{j+1}}^{*}-x_{i_{j}}^{*} \leq 2 \xi$ for all $j \in\{4, \ldots, n-4\}$; if this were not the case, players $i_{1}, i_{2}, i_{n-1}, i_{n}$, who earn $\xi$, would have an incentive to deviate anywhere in the open interval $] x_{i_{j}}^{*}, x_{i_{j+1}}^{*}\left[\right.$ and win $\left(x_{i_{j+1}}^{*}-x_{i_{j}}^{*}\right) / 2>2 \xi$.

(iv) $x_{i_{j+1}}^{*}-x_{i_{j-1}}^{*} \geq 2 \xi$ for all $j \in\{4, \ldots, n-4\}$; in fact, if $j \in\{4, \ldots, n-4\}$, then

$$
\rho_{j}\left(\boldsymbol{x}^{*}\right)=\overleftarrow{p}_{i_{j}}\left(\boldsymbol{x}^{*}\right)+\vec{p}_{i_{j}}\left(\boldsymbol{x}^{*}\right)=\frac{x_{i_{j+1}}^{*}-x_{i_{j}}^{*}}{2}+\frac{x_{i_{j}}^{*}-x_{i_{j-1}}^{*}}{2}=\frac{x_{i_{j+1}}^{*}-x_{i_{j-1}}^{*}}{2}
$$

If $\rho_{j}\left(\boldsymbol{x}^{*}\right)<\xi$ then player $j$ would deviate to $x_{1}^{*}-\varepsilon$ and win $\xi-\varepsilon$.

It is easy to check that any location $\boldsymbol{x}$ which verifies (i)-(iv) is a pure Nash equilibrium since it does not allow any profitable deviation (see Figure 8). Therefore conditions (i)-(iv) characterize equilibria in $\mathscr{H}\left(N_{n},[0,1], \boldsymbol{\rho}\right)$. Since they are linear inequalities, the set of equilibria is a polytope.

Lemma A.27. For every $\boldsymbol{x} \in[0,1]^{n}$ we have

$$
\sigma(\boldsymbol{x})=\frac{x_{1}^{2}}{2}+\sum_{i=1}^{n-1} \frac{\left(x_{j+1}-x_{j}\right)^{2}}{4}+\frac{1-x_{n}^{2}}{2}=\frac{\sum_{i=1}^{n}\left[\left(\overleftarrow{p}_{i}(\boldsymbol{x})\right)^{2}+\left(\vec{p}_{i}(\boldsymbol{x})\right)^{2}\right]}{2}
$$

Proof. This is immediate from the definition of $\sigma, \vec{p}_{i}(\boldsymbol{x})$ and $\overleftarrow{p}_{i}(\boldsymbol{x})$

Lemma A.28. If for all $i \in N_{n}$

$$
\bar{x}_{i}=\frac{2 i-1}{2 n},
$$


then

$$
\overline{\boldsymbol{x}} \in \arg \min _{\boldsymbol{x} \in[0,1]^{n}} \sigma(\boldsymbol{x})
$$

and

$$
\text { Opt } \mathscr{H}\left(N_{n}, S, \boldsymbol{\rho}\right)=\sigma(\overline{\boldsymbol{x}})=\frac{1}{4 n} .
$$

Proof. For all $i \in N_{n}$ we have

$$
\overleftarrow{p}_{i}(\overline{\boldsymbol{x}})=\vec{p}_{i}(\overline{\boldsymbol{x}})=\frac{1}{2 n}
$$

Define

$$
\mathbb{R}^{2 n} \ni \boldsymbol{p}(\boldsymbol{x}):=\left(\overleftarrow{p}_{1}(\boldsymbol{x}), \vec{p}_{1}(\boldsymbol{x}), \ldots, \overleftarrow{p}_{n}(\boldsymbol{x}), \vec{p}_{n}(\boldsymbol{x})\right)
$$

Hence

$$
\boldsymbol{p}(\overline{\boldsymbol{x}}):=\left(\frac{1}{2 n}, \ldots, \frac{1}{2 n}\right) .
$$

Then for all $\boldsymbol{x} \in[0,1]^{n}$ we have $\boldsymbol{p}(\overline{\boldsymbol{x}}) \prec \boldsymbol{p}(\boldsymbol{x})$. Hence, using Lemma A.27 and Schurconvexity of the function

$$
\boldsymbol{p}(\boldsymbol{x}) \mapsto \frac{\sum_{i=1}^{n}\left[\left(\overleftarrow{p}_{i}(\boldsymbol{x})\right)^{2}+\left(\vec{p}_{i}(\boldsymbol{x})\right)^{2}\right]}{2}
$$

for every $\boldsymbol{x} \in[0,1]^{n}$ we have

$$
\sigma(\overline{\boldsymbol{x}}) \leq \sigma(\boldsymbol{x})
$$

which proves (A.18). To prove (A.19) consider that the average cost generated by a half domain $p_{i}(\overline{\boldsymbol{x}})$ is $1 / 8 n^{2}$ and there are $2 n$ such half domains (See Figure 9 ).

Lemma A.29. Consider the game $\mathscr{H}\left(N_{n},[0,1], \boldsymbol{\rho}\right)$ and the action profiles $\check{\boldsymbol{x}}, \hat{\boldsymbol{x}}$, and $\hat{\boldsymbol{x}}^{\ell}$, defined as follows:

For every positive $n$

$$
\begin{aligned}
& \check{x}_{1}=\check{x}_{2}=\frac{1}{n-4}, \\
& \check{x}_{i}=\frac{2 i-1}{n-4}, \quad i \in\{3, \ldots, n-2\}, \\
& \check{x}_{n-1}=\check{x}_{n}=\frac{n-5}{n-4} .
\end{aligned}
$$

For $n$ even

$$
\hat{x}_{2 i-1}=\hat{x}_{2 i}=\frac{2 i-1}{n}, \quad i \in\{1, \ldots, n / 2\} .
$$

For $n$ odd and $\ell \in\left\{2, \ldots,\left\lfloor\frac{n-2}{2}\right\rfloor\right\}$

$$
\begin{array}{ll}
\hat{x}_{2 i-1}^{\ell}=\hat{x}_{2 i}^{\ell}=\frac{2 i-1}{n+1}, & i \in\{1, \ldots, \ell\}, \\
\hat{x}_{2 \ell+1}^{\ell}=\frac{2 \ell+1}{n+1}, & \\
\hat{x}_{2 i}^{\ell}=\hat{x}_{2 i+1}^{\ell}=\frac{2 i+1}{n+1}, & i \in\left\{\ell+1, \ldots,\left\lfloor\frac{n}{2}\right\rfloor\right\} .
\end{array}
$$


(a) The action profiles $\check{\boldsymbol{x}}, \hat{\boldsymbol{x}}$, and $\hat{\boldsymbol{x}}^{\ell}$ are equilibria in $\mathscr{H}\left(N_{n},[0,1], \boldsymbol{\rho}\right)$.

(b) For all $n \geq 4$

$$
\check{\boldsymbol{x}}=\arg \min _{x \in \mathscr{E}(\mathscr{H})} \sigma(\boldsymbol{x})
$$

and

$$
\text { BEq } \mathscr{H}\left(N_{n},[0,1], \boldsymbol{\rho}\right)=\sigma(\check{\boldsymbol{x}})=\frac{1}{4(n-2)}
$$

(c) For $n$ even

$$
\hat{\boldsymbol{x}}=\arg \max _{x \in \mathscr{E}(\mathscr{H})} \sigma(\boldsymbol{x})
$$

and

$$
\text { WEq } \mathscr{H}\left(N_{n},[0,1], \boldsymbol{\rho}\right)=\sigma(\hat{\boldsymbol{x}})=\frac{1}{2 n}
$$

(d) For $n>3$ odd

$$
\hat{\boldsymbol{x}}^{\ell}=\arg \max _{x \in \mathscr{E}(\mathscr{H})} \sigma(\boldsymbol{x})
$$

and

$$
\text { WEq } \mathscr{H}\left(N_{n},[0,1], \boldsymbol{\rho}\right)=\sigma\left(\hat{\boldsymbol{x}}^{\ell}\right)=\frac{n}{2(n+1)} \text {. }
$$

Proof. (a) It is easy to see that $\check{\boldsymbol{x}}, \hat{\boldsymbol{x}}, \hat{\boldsymbol{x}}^{\ell}$ are either the unique equilibria of $\mathscr{H}\left(N_{n},[0,1], \boldsymbol{\rho}\right)$ as in Proposition 4.3(a)(b) or satisfy conditions (i)-(iv) of the proof of Proposition 4.3(c).

(b) By Lemma A.26(c) for every equilibrium $\boldsymbol{x}^{*}$ of $\mathscr{H}\left(N_{n},[0,1], \boldsymbol{\rho}\right)$ there exists $\xi>0$ such that

$$
\begin{aligned}
& \overleftarrow{p}_{1}\left(\boldsymbol{x}^{*}\right)=\vec{p}_{2}\left(\boldsymbol{x}^{*}\right)=\overleftarrow{p}_{3}\left(\boldsymbol{x}^{*}\right)=\vec{p}_{n-2}\left(\boldsymbol{x}^{*}\right)=\overleftarrow{p}_{n-1}\left(\boldsymbol{x}^{*}\right)=\vec{p}_{n}\left(\boldsymbol{x}^{*}\right)=\xi \\
& \vec{p}_{1}\left(\boldsymbol{x}^{*}\right)=\overleftarrow{p}_{2}\left(\boldsymbol{x}^{*}\right)=\vec{p}_{n-1}\left(\boldsymbol{x}^{*}\right)=\overleftarrow{p}_{n}\left(\boldsymbol{x}^{*}\right)=0 \\
& \vec{p}_{i}\left(\boldsymbol{x}^{*}\right) \leq \xi, \quad \overleftarrow{p}_{i}\left(\boldsymbol{x}^{*}\right) \leq \xi, \quad \vec{p}_{i}\left(\boldsymbol{x}^{*}\right)+\overleftarrow{p}_{i}\left(\boldsymbol{x}^{*}\right) \geq \xi \quad \text { for all } i \in N_{n}
\end{aligned}
$$

Moreover

$$
\begin{gathered}
\overleftarrow{p}_{1}(\check{\boldsymbol{x}})=\vec{p}_{2}(\check{\boldsymbol{x}})=\overleftarrow{p}_{3}(\check{\boldsymbol{x}})=\vec{p}_{n-2}(\check{\boldsymbol{x}})=\overleftarrow{p}_{n-1}(\check{\boldsymbol{x}})=\vec{p}_{n}(\check{\boldsymbol{x}})=\frac{1}{2 n-4} \\
\vec{p}_{1}(\check{\boldsymbol{x}})=\overleftarrow{p}_{2}(\check{\boldsymbol{x}})=\vec{p}_{n-1}(\check{\boldsymbol{x}})=\overleftarrow{p}_{n}(\check{\boldsymbol{x}})=0
\end{gathered}
$$

and for all $i \in\{4, \ldots, n-3\}$

$$
\vec{p}_{i}(\check{\boldsymbol{x}})=\overleftarrow{p}_{i}(\check{\boldsymbol{x}})=\vec{p}_{3}(\check{\boldsymbol{x}})=\overleftarrow{p}_{n-2}(\check{\boldsymbol{x}})=\frac{1}{2 n-4}
$$


Define the simplex

$$
\mathscr{S}=\left\{\boldsymbol{q}=\left(q_{1}, \ldots, q_{2 n}\right): q_{i} \geq 0 \text { for } i=1, \ldots, 2 n, \text { and } \sum_{i=1}^{2 n} q_{i}=1\right\}
$$

and consider the vectors $\boldsymbol{q}_{1}, \boldsymbol{q}_{2} \in \mathscr{S}$, where

$$
\begin{aligned}
& \boldsymbol{q}_{1}:=\left(\frac{1}{2 n-4}, \ldots, \frac{1}{2 n-4}, 0,0,0,0\right), \\
& \boldsymbol{q}_{2}:=\left(\xi, \xi, \xi, \xi, \xi, \xi, y_{1}, \ldots, y_{2 n-10}, 0,0,0,0\right),
\end{aligned}
$$

with $\xi \geq y_{i}$ for $i=1, \ldots, 2 n-10$. Since $\boldsymbol{q}_{1}, \boldsymbol{q}_{2} \in \mathscr{S}$, we have

$$
\xi \geq \frac{1}{2 n-4} \text {. }
$$

It is not difficult to see that, using the notation of (A.17), the vector $\boldsymbol{p}(\check{\boldsymbol{x}})$ is a permutation of $\boldsymbol{q}_{1}$ and the vector $\boldsymbol{p}\left(\boldsymbol{x}^{*}\right)$ is a permutation of $\boldsymbol{q}_{2}$. Moreover $\boldsymbol{q}_{1} \prec \boldsymbol{q}_{2}$, hence $\boldsymbol{p}(\check{\boldsymbol{x}}) \prec \boldsymbol{p}\left(\boldsymbol{x}^{*}\right)$. Lemma A.27 and the usual Schur-convexity argument provide therefore (A.21).

To prove (A.22) consider that the cost generated by $2 n-4$ of the half domains $p_{i}(\check{\boldsymbol{x}})$ is

$$
(2 n-4) \frac{1}{2(2 n-4)^{2}}=\frac{1}{4 n-8}
$$

and the cost generated by the remaining 4 half domains is 0 (See Figure 10).

(c) For $i$ odd

$$
\overleftarrow{p}_{i}(\hat{\boldsymbol{x}})=\frac{1}{n}, \quad \vec{p}_{i}(\hat{\boldsymbol{x}})=0
$$

and for $i$ even

$$
\vec{p}_{i}(\hat{\boldsymbol{x}})=\frac{1}{n}, \quad \overleftarrow{p}_{i}(\hat{\boldsymbol{x}})=0
$$

Consider the vector

$$
\boldsymbol{q}_{3}:=\left(\frac{1}{n}, \ldots, \frac{1}{n}, 0, \ldots, 0\right) \in \mathscr{S} .
$$

Conditions (A.27) imply $\xi \leq 1 / n$. Therefore $\boldsymbol{q}_{2} \prec \boldsymbol{q}_{3}$. Since $\boldsymbol{q}_{3}$ is a permutation of $\hat{\boldsymbol{x}}$, we have $\boldsymbol{p}\left(\boldsymbol{x}^{*}\right) \prec \boldsymbol{p}(\hat{\boldsymbol{x}})$. Again Lemma A.27 and the usual Schur-convexity argument provide (A.23).

To prove (A.24) consider that the total average cost generated by $n$ of the half domains $p_{i}(\hat{\boldsymbol{x}})$ is $1 / 2 n^{2}$ and the average cost generated by the remaining $n$ half domains is 0 (See Figure 11).

(d) The argument is similar to the one used to prove (c) (See Figure 12).

Proof of Proposition 4.4. (a) Just combine Lemma A.28 and Lemma A.29(c)(d).

(b) Combine Lemma A.28 and Lemma A.29(b). 


\section{Proofs of Subsection 4.3}

For $j \in\{1, \ldots, k\}$ define

$$
N^{j}=\left\{i \in N: x_{i} \in e_{v_{0} v_{j}} \backslash\left\{v_{0}\right\}\right\}
$$

and call $h(j)$ the cardinality of $N^{j}$. Order the players $i_{j, 1} \prec \cdots \prec i_{j, h(j)} \in N^{j}$ in terms of the distance of their actions $x_{i_{j, 1}}, \ldots, x_{i_{j, h(j)}}$ from $v_{0}$, from the smallest to the largest (solve the ties arbitrarily).

For $j \in\{1, \ldots, k\}$, define

$$
p_{j}(\boldsymbol{x})= \begin{cases}d\left(v_{0}, x_{i_{j, 1}}\right) / 2 & \text { if there is at least one player on } e_{v_{0} v_{j}} \\ 1 & \text { otherwise. }\end{cases}
$$

Call $g_{i_{j, \ell}}$ the payoff of player $i_{j, \ell}$.

Lemma A.30. If the profile $\boldsymbol{x}$ is such that there is a player in $v_{0}$, then

(a) the payoff of the player in $v_{0}$ is

$$
\frac{\sum_{j=1}^{k} p_{j}(\boldsymbol{x})}{\operatorname{card}\left\{\ell: x_{l}=v_{0}\right\}}
$$

(b) for $j$ in $\{1, \ldots, k\}$ we have

$$
\begin{aligned}
g_{i_{j, 1}} & =\frac{d\left(v_{0}, x_{i_{j, 1}}\right)+d\left(x_{i_{j, 1}}, x_{i_{j, 2}}\right)}{2 \operatorname{card}\left\{m: x_{m}=x_{i_{j, 1}}\right\}}, \\
g_{i_{j, \ell}} & =\frac{d\left(x_{i_{j, \ell-1}}, x_{i_{j, \ell}}\right)+d\left(x_{i_{j, \ell}}, x_{i_{j, \ell+1}}\right)}{2 \operatorname{card}\left\{m: x_{m}=x_{i_{j, \ell}}\right\}} \text { for } \ell \in\{2, \ldots, h(j)-1\}, \\
g_{i_{j, h(j)}} & =\frac{2 d\left(x_{i_{j, h(j)}}, v_{j}\right)+d\left(x_{i_{j, h(j)}}, x_{i_{j, h(j)-1}}\right)}{2 \operatorname{card}\left\{m: x_{m}=x_{i_{j, h(j)}}\right\}}
\end{aligned}
$$

Proof. Obvious from the definition

Lemma A.31. Let $\bar{j}=\arg \min _{j} p_{j}$ and assume that in the profile $\boldsymbol{x}$ there is no player in $v_{0}$. Then for $j$ in $\{1, \ldots, k\}$ we have

$$
g_{i_{j, 1}}= \begin{cases}\frac{d\left(x_{i_{j, 1}}, x_{i_{j}, 2}\right)+d\left(x_{i_{j, 1}}, x_{i_{\bar{j}, 1}}\right)}{2 \operatorname{card}\left\{m: x_{m}=x_{i_{j, 1}}\right\}} & \text { for } j \neq \bar{j} \\ \frac{2 p_{\bar{j}}+\sum_{j \neq \bar{j}}\left(p_{j}-p_{\bar{j}}\right)+d\left(x_{i_{\bar{j}, 1}}, x_{i_{\bar{j}, 2}}\right)}{2 \operatorname{card}\left\{m: x_{m}=x_{i_{\bar{j}, 1}}\right\}} & \text { for } j=\bar{j}\end{cases}
$$

with $x_{i_{j, 2}}=v_{j}$ if $x_{i_{j, 1}}$ is the only location on the edge $j$. 
Proof. Obvious from the definition

Lemma A.32. Let $\boldsymbol{x}^{*}$ be a Nash equilibrium of the Hotelling game $\mathscr{H}\left(N_{n}, S_{k}, \boldsymbol{\rho}\right)$. Then the following hold:

(a) There exists $i^{*} \in\{1, \ldots, N\}$ such that $x_{i^{*}}^{*}=v_{0}$.

(b) For every $x \in S \backslash\left\{v_{0}\right\}, \operatorname{card}\left\{i: x_{i}^{*}=x\right\} \leq 2$.

(c) $\operatorname{card}\left\{i: x_{i}^{*}=v_{0}\right\} \leq k$.

(d) If for some $i \in N_{n}$ we have $x_{i}^{*} \in e_{v_{0} v_{j}}$, then for each $j \in\{1, \ldots, k\}$ there exist at least two players $i_{j, h(j)}, i_{j, h(j)-1} \in N^{j}$ such that $x_{i_{j, h(j)}^{*}}^{*}=x_{i_{j, h(j)-1}^{*}}^{*}$.

Proof. (a) Consider a star $S_{k}$ with $k \geq 3$ and assume ad absurdum that no player is in $v_{0}$. Consider the player $i$ such that $x_{i}^{*}$ is the closest position to $v_{0}$. For $\varepsilon<d\left(x_{i}^{*}, v_{0}\right)$, if player $i$ moves of $\varepsilon$ towards the center, then she loses $\varepsilon / 2$ on the edge where she is and gains $\varepsilon / 2$ on every other edge. Therefore moving towards the center is profitable. (b) Since $x^{*} \in S \backslash\left\{v_{0}\right\}$ and since there is a player in $v_{0}$, we are in the situation considered in Lemma A.26(b). Therefore, at most two players can be in the same location.

(c) Suppose that $\operatorname{card}\left\{i: x_{i}^{*}=v_{0}\right\}=k^{\prime}>k$. Then the players in the center win

$$
\frac{1}{k^{\prime}} \sum_{j=0}^{k} p_{j}\left(\boldsymbol{x}^{*}\right)<\max _{j \in\{1, \ldots, k\}} p_{j}\left(\boldsymbol{x}^{*}\right) .
$$

Then a player who moves by $\varepsilon$ from $v_{0}$ in the direction of the maximum $p_{j}\left(\boldsymbol{x}^{*}\right)$ enjoys a profitable deviation.

(d) Suppose that there exists $i \in N_{n}$ such that $x_{i}^{*} \in e_{v_{0} v_{j}}$. Then player $i$ cannot be alone on her edge: if she were, she would have a profitable deviation by moving towards the center. If one edge were empty, then any of the players could profitably deviate by moving to the empty edge, close enough to $v_{0}$.

Lemma A.33. Let $\boldsymbol{x}^{*}$ be a Nash equilibrium of $\mathscr{H}\left(N_{n}, S_{k}, \boldsymbol{\rho}\right)$ and let $y \in e_{v_{0}, v_{j}} \backslash\left\{v_{0}\right\}$, be such that

$$
\operatorname{card}\left\{\ell: x_{\ell}^{*}=y\right\}=2 \text {, }
$$

and call $i_{\ell}$ and $i_{\ell+1}$ the two players in $y$. We have

(a) if $h(j)>\ell+1$, then

$$
g_{i_{j, \ell}}=g_{i_{j, \ell+1}}=d\left(x_{i_{j, \ell}}^{*}, x_{i_{j, \ell-1}}^{*}\right)=d\left(x_{i_{j, \ell+1}}^{*}, x_{i_{j, \ell+2}}^{*}\right)=: \xi(y) .
$$

(b) if $h(j)=\ell+1$, then

$$
g_{i_{j, \ell}}=g_{i_{j, \ell+1}}=d\left(x_{i_{j, \ell}}^{*}, x_{i_{j, \ell-1}}^{*}\right)=\frac{1}{2} d\left(x_{i_{j, \ell+1}}^{*}, v_{j}\right)=: \xi(y) .
$$


(c) The value $\xi(y)$ does not depend on y (hence we simply denote it $\xi$ ).

Proof. For (a) and (b) the proof is similar to the one given for Lemma A.26 for the interval.

(c) Suppose that $\xi\left(y_{1}\right)>\xi\left(y_{2}\right)$, then players in $y_{2}$ have a payoff strictly smaller than players in $y_{1}$. Then, for $\varepsilon>0$ small enough, they can profitably deviate by moving $\varepsilon$ close to $y_{1}$ and getting $\xi\left(y_{1}\right)-\varepsilon$.

Lemma A.34. Let $\boldsymbol{x}^{*}$ be a Nash equilibrium of $\mathscr{H}\left(N_{n}, S_{k}, \boldsymbol{\rho}\right)$. If $\operatorname{card}\left\{\ell: x_{\ell}^{*}=v_{0}\right\}=$ degree $\left(v_{0}\right)=k$, then

$$
p_{j}\left(\boldsymbol{x}^{*}\right)=\xi
$$

Proof. Suppose that card $\left\{\ell: x_{\ell}^{*}=v_{0}\right\}=k$, then the payoff of the players in $v_{0}$ is $k^{-1} \sum_{j=1}^{k} p_{j}(\boldsymbol{x})$.

First we prove that all the $p_{j}$ are equal, for $j \in\{1, \ldots, k\}$. Indeed suppose, ad absurdum, that

$$
\max _{j} p_{j}\left(\boldsymbol{x}^{*}\right)>\frac{\sum_{j=1}^{k} p_{j}(\boldsymbol{x})}{k} .
$$

If $\hat{j} \in \arg \max _{j} p_{j}(\boldsymbol{x})$, then any player in the center would improve her payoff by moving towards $v_{\hat{j}}$ by $\varepsilon$ small enough.

Now we have to prove that

$$
\frac{\sum_{j=1}^{k} p_{j}(\boldsymbol{x})}{k}=\xi
$$

Suppose, ad absurdum, that $\xi>k^{-1} \sum_{j=1}^{k} p_{j}(\boldsymbol{x})$ and that $y$ satisfies the conditions of Lemma A.33. Then any player in the center could profitably deviate by moving very close to $y$ and increase her payoff up to $\xi-\varepsilon$, for any $\varepsilon>0$. Suppose now that $\xi<k^{-1} \sum_{j=1}^{k} p_{j}(\boldsymbol{x})$. Then one of the two players in $y$ can move very close to $v_{0}$ on any edge and increase his payoff up to $k^{-1} \sum_{j=1}^{k} p_{j}(\boldsymbol{x})-\varepsilon$ for any $\varepsilon>0$. Therefore in equilibrium (A.32) holds.

Proof of Proposition 4.5. (a) First we prove that the profile $\boldsymbol{x}^{*}$ such that $x_{i}^{*}=v_{0}$ for all $i \in N_{n}$ is indeed an equilibrium. If any player $i$ deviates, he will obtain a payoff that is strictly less than 1 , whereas by not deviating she obtains $k / n \geq 1$.

Now we turn to prove uniqueness. Assume by contradiction that there exists an equilibrium such that for some $i \in N_{n}$ we have $x_{i} \in e_{v_{0} v_{j}}$. Then, by Lemma A.32(d), each edge $e_{v_{0} v_{j}}$ has been chosen by at least two players. This implies that $n \geq 2 k$, which is impossible, since $n \leq k$.

(b) Assume by contradiction that an equilibrium $\boldsymbol{x}^{*}$ exists. If $x_{i}^{*}=v_{0}$ for all $i \in N_{n}$, then each player gains $k / n \leq 1$, so a profitable deviation is possible.

We consider now the case where for some $i \in N_{n}$ we have $x_{i} \neq v_{0}$. 
First we consider the case $k<n<2 k$. If for some $i \in N_{n}$ we have $x_{i}^{*} \neq v_{0}$, then, by Lemma A.32(d), $2 k$ players choose an action different from $v_{0}$, which is impossible since $n<2 k$.

If $n=2 k$, and for some $i \in N_{n}$ we have $x_{i}^{*} \neq v_{0}$, then, by Lemma A.32(d), $x_{i}^{*} \neq v_{0}$ for all $i \in N_{n}$ and, by Lemma A.33, $d\left(x_{i}^{*}, v_{0}\right)=1 / 2$. This cannot be an equilibrium, since any player $i$ has an incentive to deviate choosing $x_{i}^{*}=v_{0}$.

Assume now $2 k<n<3 k-1$. The profile where all the players choose $v_{0}$ is not an equilibrium. By Lemma A.32(d), for each $j \in\{1, \ldots, k\}$, there exist at least two players $i_{j, h(j)-1}, i_{j, h(j)} \in N^{j}$ such that $d\left(x_{i_{j, h(j)-1}^{*}}^{*}, v_{j}\right)=d\left(x_{i_{j, h(j)}}^{*}, v_{j}\right)=\xi$. The equilibrium action of the remaining $n-2 k$ players must be $v_{0}$. If this were not the case, then, for some $j \in\{1, \ldots, k\}$, there would be three players on the edge $j$ and

$$
1 \leq d\left(v_{0}, x_{j, 1}^{*}\right)+d\left(x_{j, 1}^{*}, x_{j, h(j)}^{*}\right)+d\left(x_{j, h(j)}^{*}, v_{j}\right)=d\left(v_{0}, x_{j, 1}^{*}\right)+3 \xi .
$$

On the other hand, there are at most $k-2$ remaining players, so there is an other edge with only two players. This implies $1=3 \xi$, which is a contradiction. Therefore the remaining $k-2$ players left must be in $v_{0}$. The payoff of every player $i$ such that $x_{i}^{*}=v_{0}$ equals $k / 3(n-2 k)$. The payoff of every other player is $1 / 3$ and therefore any of them would have an incentive to deviate to $v_{0}$, gaining $k / 3(n-2 k+1)$ which is larger than $1 / 3$ when $n<3 k-1$.

(c) It is easy to prove that a profile $\boldsymbol{x}^{*}$ where on each edge two players sit at a distance $2 / 3$ from the origin and the remaining players sit at $v_{0}$ is an equilibrium. We now show uniqueness. Indeed we know from Lemma A.32(c) that a profile where all players choose $v_{0}$ is not an equilibrium; moreover Lemma A.32(d) implies that each edge has at least two players. Using the same argument that we used in the proof of (b), we can show that no edge can have three players if another edge has only two. By Lemma A.32(a), at least one player chooses $v_{0}$, therefore it is not possible to have three players on each edge, if $n \in\{3 k-1,3 k\}$. Hence all remaining players are in $v_{0}$. (d) We now assume $n \geq 3 k+1$. Let $n=m k+r$ be the Euclidean division of $n$ by $k$. We will construct an equilibrium $\boldsymbol{x}^{*}$ with $m$ players on each edge and $r$ players in the center, like in Figure 15. Let $(2 m-3) \xi+y=1$. This profile is indeed an equilibrium if and only if the following conditions are satisfied:

(i) None of the $r$ players in $v_{0}$ has an incentive to deviate to an interval of length $2 \xi$, that is, for all $i \in N_{n}$ such that $x_{i}^{*}=v_{0}$, we have $\rho_{i}\left(\boldsymbol{x}^{*}\right)=k y / 2 r \geq \xi$, which implies $y \geq 2 r \xi / k$.

(ii) No player has an incentive to deviate to $v_{0}$. Given that $\rho_{i}\left(\boldsymbol{x}^{*}\right) \geq \xi$ for all $i \in N_{n}$ such that $x_{i}^{*} \neq v_{0}$, we have $k y / 2(r+1) \leq \xi$, which implies $y \leq 2(r+1) \xi / k$.

(iii) No player has an incentive to deviate to an interval of length $y$, that is $y \leq 2 \xi$.

(iv) No player has an incentive to deviate to a location with another single player. 
If she did, her payoff would be either $\xi$ or

$$
\frac{\xi}{2}+\frac{y}{4} \leq \xi
$$

Then, for any $\xi$ such that

$$
\frac{k}{2(r+1)+2 k m-3 k} \leq \xi \leq \frac{k}{2 r+2 k m-3 k}
$$

the profile $\boldsymbol{x}^{*}$ is an equilibrium. Hence we have an infinite number of Nash pure equilibria.

\section{References}

Anderson, S. P., Goeree, J. K., and Ramer, R. (1997) Location, location, location. J. Econom. Theory 77, 102-127.

Anderson, S. P., De Palma, A., and Thisse, J.-F. (1992) Discrete Choice Theory of Product Differentiation. MIT Press, Cambridge, MA.

Aoyagi, M. and Okabe, A. (1993) Spatial competition of firms in a two-dimensional bounded market. Regional Sci. Urban Econ. 23, 259-289.

Bester, H., de Palma, A., Leininger, W., Thomas, J., and von ThadDEN, E.-L. (1996) A noncooperative analysis of Hotelling's location game. Games Econom. Behav. 12, 165-186.

Brenner, S. (2005) Hotelling games with three, four, and more players. Journal of Regional Science 45, 851-864.

Brenner, S. (2010) Location (Hotelling) games and applications. In Cochran, J. J. (ed.), Wiley Encyclopedia of Operations Research and Management Science. John Wiley \& Sons, Inc.

Chamberlin, E. H. (1933) The Theory of Monopolistic Competition. Harvard University Press, Cambridge, Mass.

Correa, J. R., Schulz, A. S., and Stier-Moses, N. E. (2004) Selfish routing in capacitated networks. Math. Oper. Res. 29, 961-976.

Correa, J. R., Schulz, A. S., and Stier-Moses, N. E. (2007) Fast, fair, and efficient flows in networks. Oper. Res. 55, 215-225. 
Correa, J. R., Schulz, A. S., and Stier-Moses, N. E. (2008) A geometric approach to the price of anarchy in nonatomic congestion games. Games Econom. Behav. 64, 457-469.

D'Aspremont, C., Gabszewicz, J. J., and Thisse, J.-F. (1979) On Hotelling's "Stability in competition". Econometrica 47, 1145-1150.

Eaton, B. C. and Lipsey, R. G. (1975) The principle of minimum differentiation reconsidered: some new developments in the theory of spatial competition. Rev. Econ. Stud. 42, 27-49.

ECONOMides, N. (1993) Hotelling's "Main Street" with more than two competitors. J. Regional Sci. 33, 303-319.

Eiselt, H. A. and Laporte, G. (1993) The existence of equilibria in the 3-facility Hotelling model in a tree. Transportation Sci. 27, 39-43.

Epstein, A., Feldman, M., and Mansour, Y. (2009) Strong equilibrium in cost sharing connection games. Games Econom. Behav. 67, 51-68.

Feldmann, R., Mavronicolas, M., and Monien, B. (2009) Nash equilibria for Voronoi games on transitive graphs. In LEONARDI, S. (ed.), Internet and Network Economics, volume 5929 of Lecture Notes in Computer Science, 280-291. Springer Berlin Heidelberg.

Hoefer, M. (2011) Competitive cost sharing with economies of scale. Algorithmica 60, 743-765.

Hotelling, H. (1929) Stability in competition. Econ. J. 39, 41-57.

Huck, S., Müller, W., and VRIEnd, N. J. (2002) The East end, the West end, and King's Cross: on clustering in the four-player Hotelling game. Economic Inquiry 40, 231-240.

Immorlica, N., Markakis, E., and Piliouras, G. (2013) Coalition formation and price of anarchy in Cournot oligopolies. Mimeo.

Irmen, A. and Thisse, J.-F. (1998) Competition in multi-characteristics spaces: Hotelling was almost right. J. Econom. Theory 78, 76-102.

Knight, V. A. and Harper, P. R. (2013) Selfish routing in public services. European J. Oper. Res. 230, 122-132.

Koutsoupias, E. and Papadimitriou, C. (1999) Worst-case equilibria. In STACS 99 (Trier), volume 1563 of Lecture Notes in Comput. Sci., 404-413. Springer, Berlin. 
Lederer, P. J. and Hurter, JR., A. P. (1986) Competition of firms: discriminatory pricing and location. Econometrica 54, 623-640.

Lerner, A. P. and Singer, H. W. (1937) Some notes on duopoly and spatial competition. J. Polit. Econ. 45, 145-186.

Marshall, A. W., Olkin, I., and Arnold, B. C. (2011) Inequalities: Theory of Majorization and its Applications. Springer Series in Statistics. Springer, New York, second edition.

Mavronicolas, M., Monien, B., Papadopoulou, V. G., and Schoppmann, F. (2008) Voronoi games on cycle graphs. In Mathematical Foundations of Computer Science 2008, volume 5162 of Lecture Notes in Comput. Science, 503-514. Springer, Berlin.

Moulin, H. (2008) The price of anarchy of serial, average and incremental cost sharing. Econom. Theory 36, 379-405.

Moulin, H. (2010) Auctioning or assigning an object: some remarkable VCG mechanisms. Soc. Choice Welf. 34, 193-216.

von Neumann, J. (1928) Zur Theorie der Gesellschaftsspiele. Math. Ann. 100, $295-320$.

Osborne, M. J. and Pitchik, C. (1986) The nature of equilibrium in a location model. Internat. Econom. Rev. 27, 223-237.

Osborne, M. J. and Pitchik, C. (1987) Equilibrium in Hotelling's model of spatial competition. Econometrica 55, 911-922.

de Palma, A., Ginsburgh, V., Papageorgiou, Y. Y., and Thisse, J.-F. (1985) The principle of minimum differentiation holds under sufficient heterogeneity. Econometrica 53, 767-781.

PÁlvÖLGYi, D. (2011) Hotelling on graphs. Mimeo.

Papadimitriou, C. (2001) Algorithms, games, and the internet. In Proceedings of the Thirty-Third Annual ACM Symposium on Theory of Computing, 749-753. ACM, New York.

Roughgarden, T. (2012) The price of anarchy in games of incomplete information. In Proceedings of the 13th ACM Conference on Electronic Commerce, EC '12, 862879. ACM, New York, NY, USA.

Roughgarden, T. and Tardos, É. (2002) How bad is selfish routing? J. ACM 49, 236-259 (electronic). 
Roughgarden, T. and TArdos, É. (2004) Bounding the inefficiency of equilibria in nonatomic congestion games. Games Econom. Behav. 47, 389-403.

Salop, S. C. (1979) Monopolistic competition with outside goods. Bell J. Econ. 10, $141-156$.

Schulz, A. S. and Stier Moses, N. (2003) On the performance of user equilibria in traffic networks. In Proceedings of the Fourteenth Annual ACM-SIAM Symposium on Discrete Algorithms, SODA '03, 86-87. Society for Industrial and Applied Mathematics, Philadelphia, PA, USA.

Soetevent, A. R. (2010) Price competition on graphs. Technical Report Discussion Paper 10-126/1, Tinbergen Institute. 


\section{B Figures}

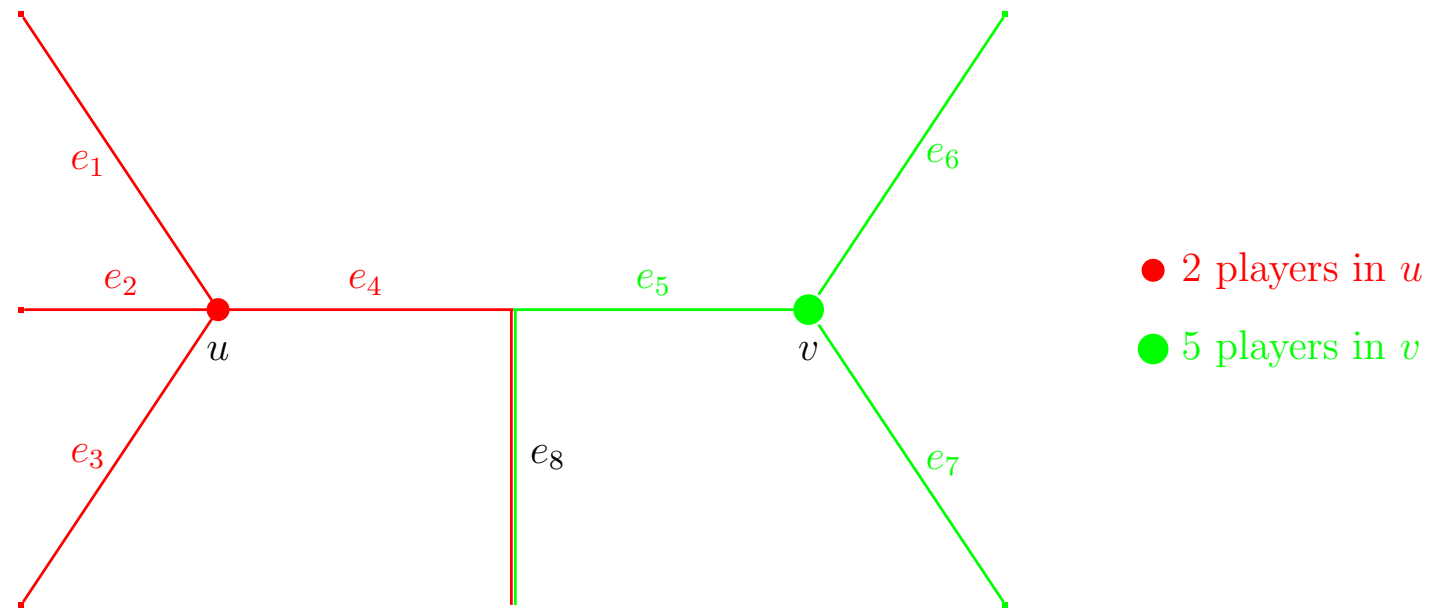

Figure 1: Domains of attraction when $\lambda\left(e_{4}\right)=\lambda\left(e_{5}\right)$. 


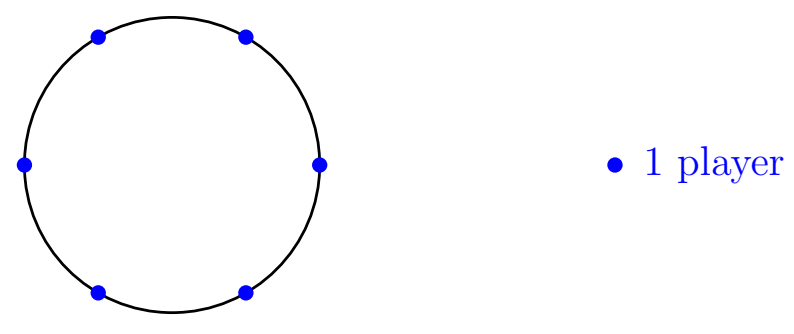

Figure 2: Best equilibrium $\tilde{\boldsymbol{x}}$ on $C$ with 6 players.

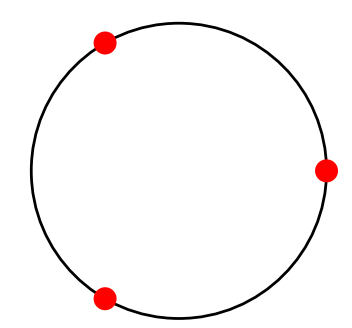

- 2 players

Figure 3: Worst equilibrium $\tilde{\tilde{\boldsymbol{x}}}$ on $C$ with 6 players.

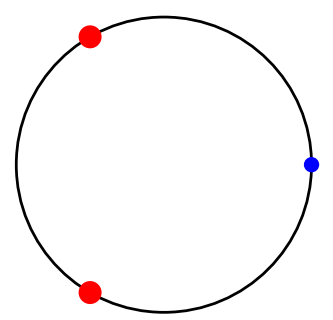

- 1 player

- 2 players

Figure 4: Worst equilibrium $\tilde{\tilde{\boldsymbol{x}}}^{\perp}$ on $C$ with 5 players. 


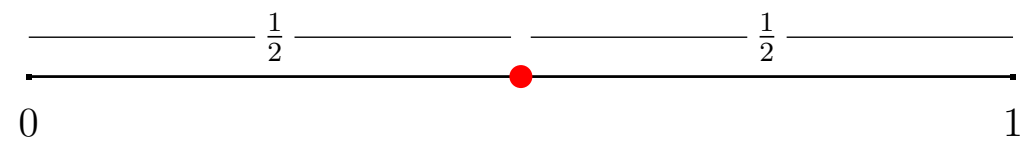

- 2 players

Figure 5: Unique equilibrium with 2 players.

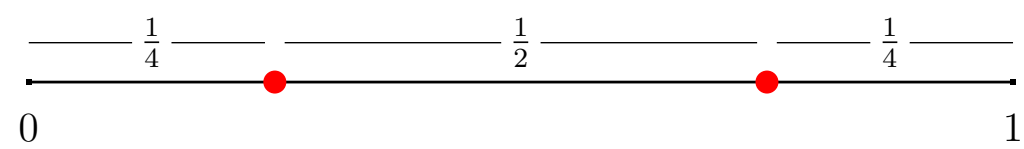

- 2 players

Figure 6: Unique equilibrium with 4 players.

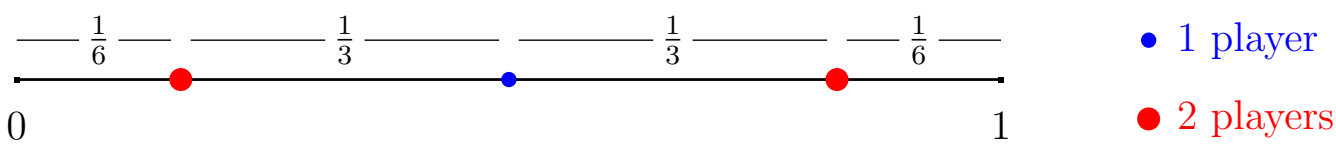

Figure 7: Unique equilibrium with 5 players.

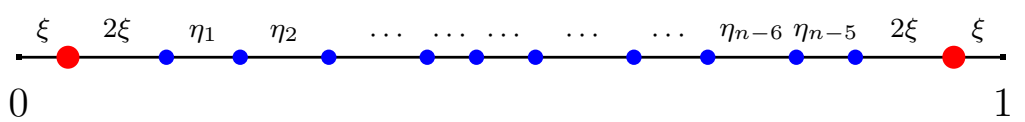

- 1 player

- 2 players

Figure 8: Example of equilibrium with $n$ players. 


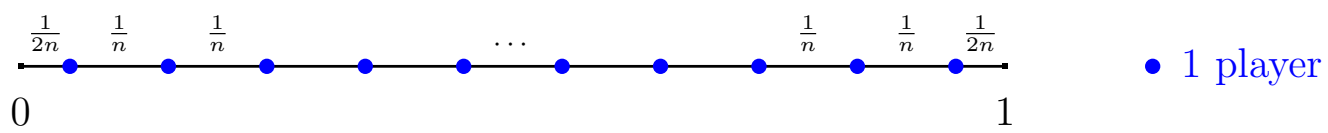

Figure 9: Social optimum $\overline{\boldsymbol{x}}$ with $n$ players.

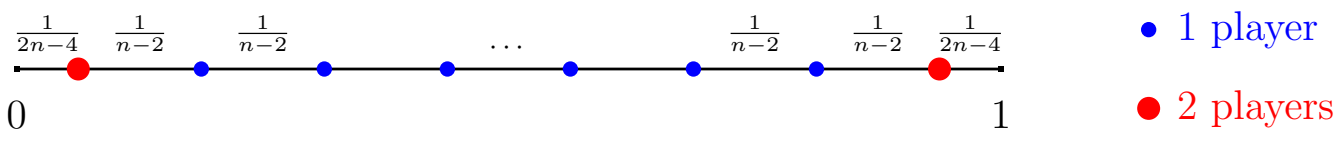

Figure 10: Best equilibrium $\check{\boldsymbol{x}}$ with $n$ players.

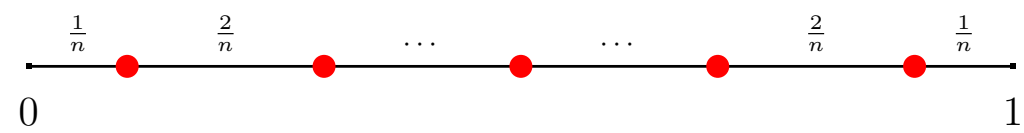

- 2 players

Figure 11: Worst equilibrium $\hat{\boldsymbol{x}}$ with $n$ players ( $n$ even).

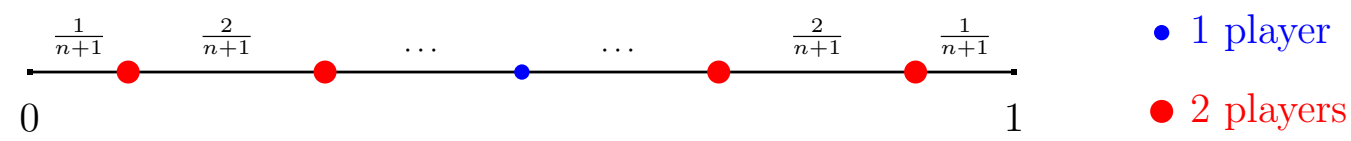

Figure 12: Worst equilibrium $\hat{\boldsymbol{x}}^{\ell}$ with $n$ players $(n$ odd, $\ell=2$ ). 


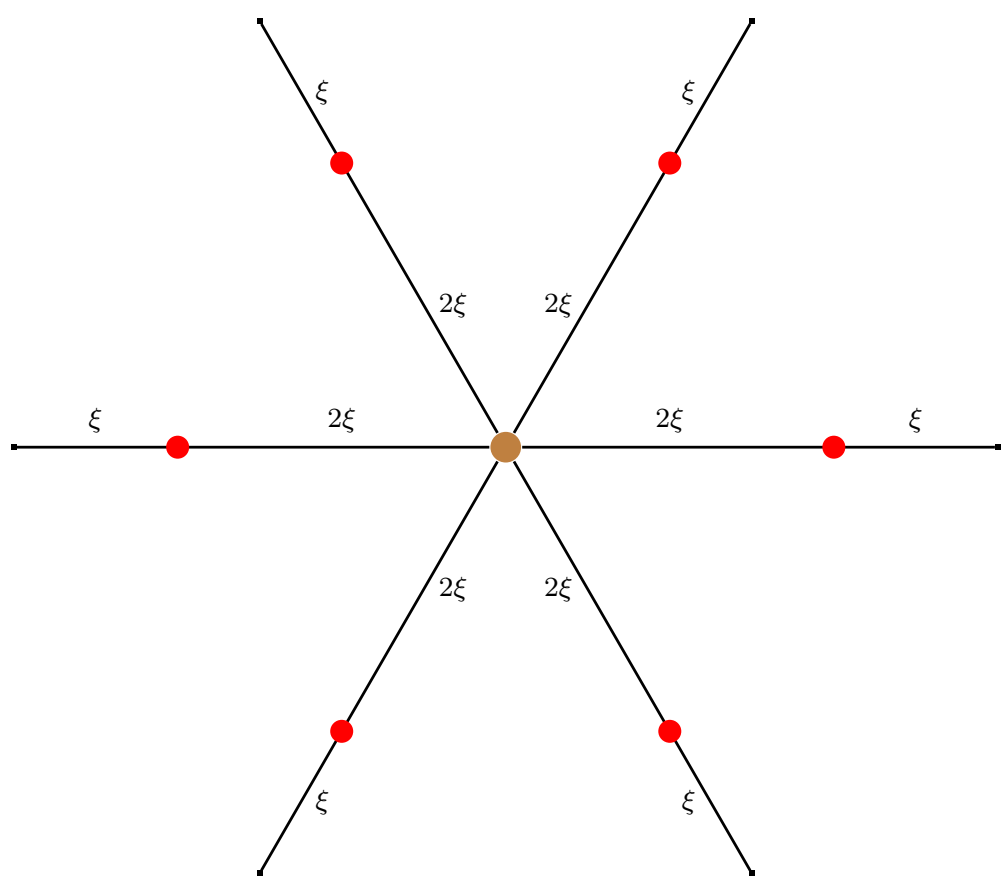

- 2 players

$k-1$ players

Figure 13: Equilibrium on $S_{k}$ with $3 k-1$ players, $k=6$.

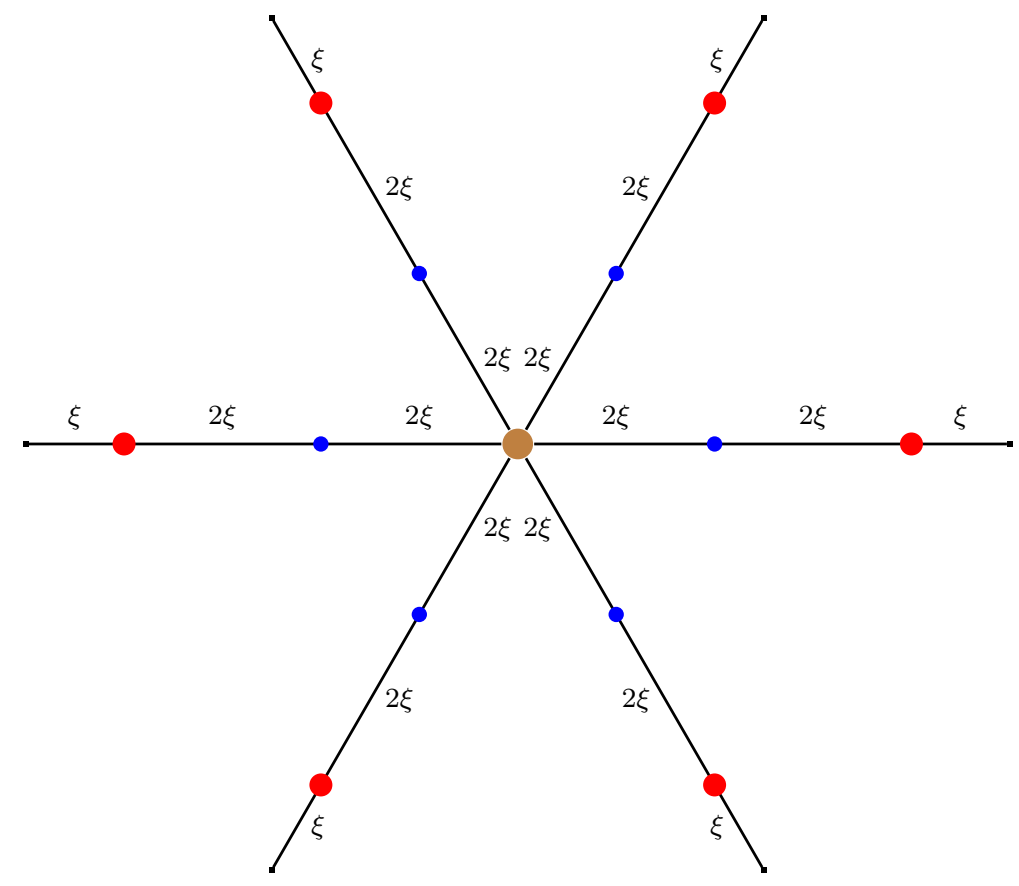

- 1 player

- 2 players

$k-1$ players

Figure 14: Equilibrium on $S_{k}$ with $4 k-1$ players, $k=6$. 


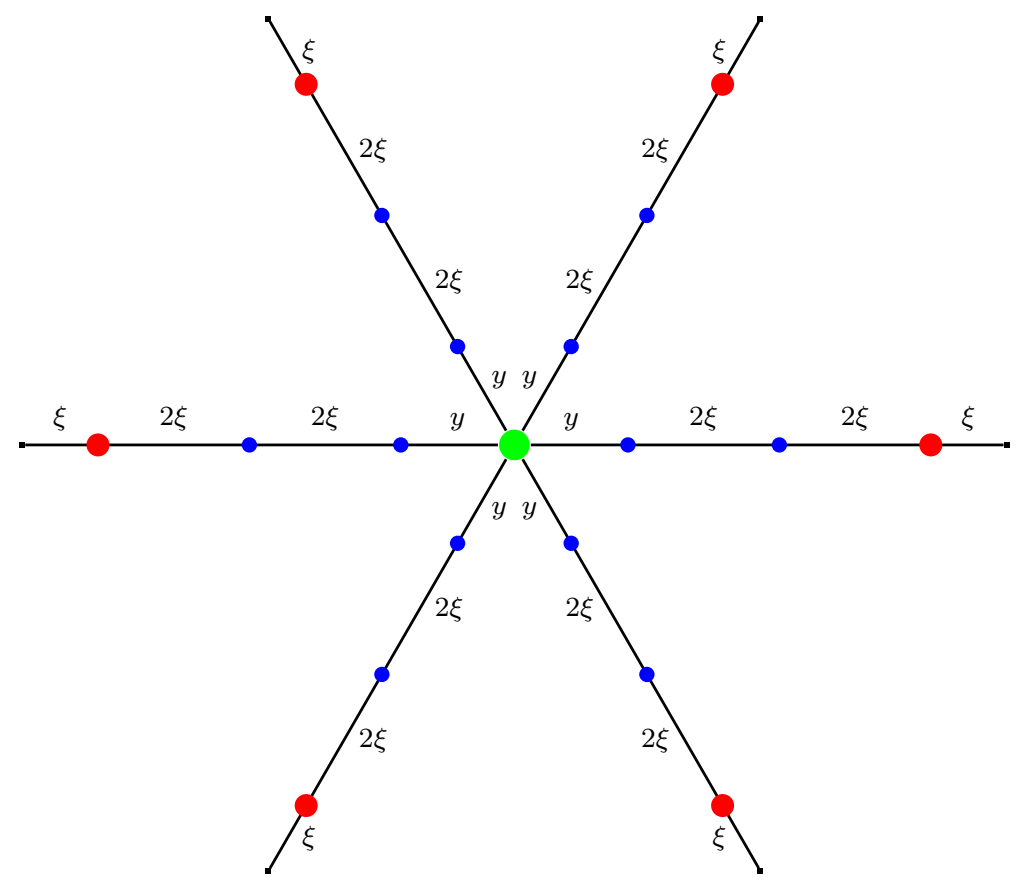

- 1 player

- 2 players

- $r$ players

Figure 15: Equilibrium on $S_{k}$ with $4 k+r$ players, $k=6(2 r \xi / k \leq y \leq 2(r+1) \xi / k)$. 


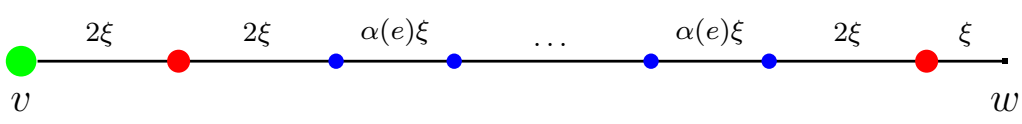

Figure 16: Players in $e \in E_{L}$.

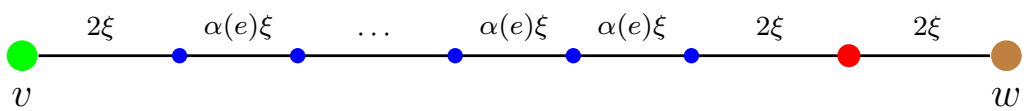

Figure 17: Players in $e \in E_{I}$.

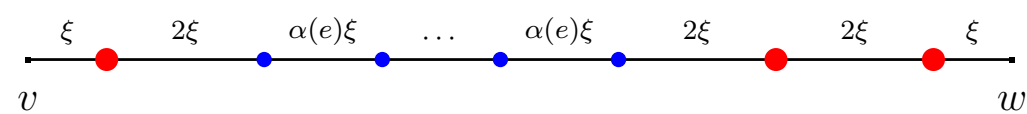

- 1 player

- 2 players

Figure 18: Players in $e \in E_{L L}$. 